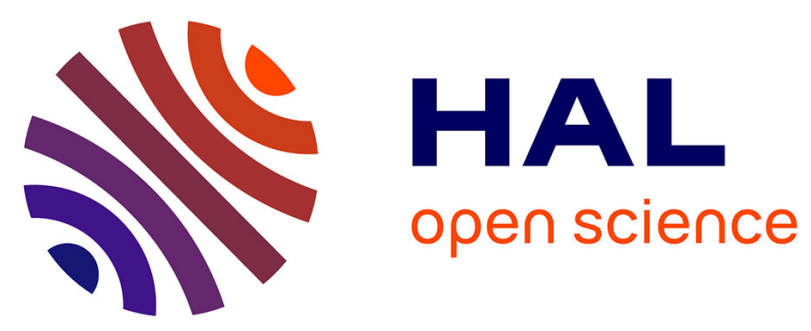

\title{
Compressible effects modelling in turbulent cavitating flows
}

\author{
Jean Decaix, Eric Goncalvès da Silva
}

\section{To cite this version:}

Jean Decaix, Eric Goncalvès da Silva. Compressible effects modelling in turbulent cavitating flows. European Journal of Mechanics - B/Fluids, 2013, 39, pp.11-31. 10.1016/j.euromechflu.2012.12.001. hal-00768757

\section{HAL Id: hal-00768757 https://hal.science/hal-00768757}

Submitted on 12 Feb 2013

HAL is a multi-disciplinary open access archive for the deposit and dissemination of scientific research documents, whether they are published or not. The documents may come from teaching and research institutions in France or abroad, or from public or private research centers.
L'archive ouverte pluridisciplinaire HAL, est destinée au dépôt et à la diffusion de documents scientifiques de niveau recherche, publiés ou non, émanant des établissements d'enseignement et de recherche français ou étrangers, des laboratoires publics ou privés. 


\title{
Compressible Effects Modelling in Turbulent Cavitating Flows
}

\author{
Jean Decaix*, Eric Goncalvès* \\ LEGI-Grenoble INP, 1025 rue de la Piscine, 38400 St Martin d'Heres, France
}

\begin{abstract}
A compressible, multiphase, one-fluid RANS solver has been developed to study turbulent cavitating flows. The interplay between turbulence and cavitation regarding the unsteadiness and structure of the flow is complex and not well understood. This constitutes a determinant point to accurately simulate the dynamic behaviour of sheet cavities. In the present study, different formulations including compressibility effects on turbulence are investigated. Numerical results are given for two partial cavities on Venturi geometries and comparisons are made with experimental data.
\end{abstract}

Keywords: Cavitation, Homogeneous model, Compressibility effects and Turbulence model

\section{Nomenclature}

$c \quad$ speed of sound

$C_{p}, C_{v} \quad$ thermal capacities

E total energy

\footnotetext{
*Corresponding author.

Email address: jean.decaix@legi.grenoble-inp.fr (Jean Decaix )
} 


\begin{tabular}{|c|c|}
\hline$e$ & internal energy \\
\hline$k$ & turbulent kinetic energy \\
\hline$M_{t}$ & turbulent Mach number \\
\hline$P$ & static pressure \\
\hline$P_{k}$ & turbulent production \\
\hline$P_{v a p}$ & vapour pressure \\
\hline$P_{r}, P_{r t}$ & molecular and turbulent Prandtl numbers \\
\hline$Q$ & total heat flux \\
\hline$R e_{L}$ & Reynolds number based on the length L \\
\hline$T$ & temperature \\
\hline$u, v$ & velocity components \\
\hline$w$ & conservative variables \\
\hline$\alpha$ & void ratio \\
\hline$\gamma$ & ratio of thermal capacities \\
\hline$\varepsilon$ & dissipation rate \\
\hline$\lambda, \lambda_{t}$ & molecular and turbulent thermal conductivity \\
\hline$\mu, \mu_{t}$ & molecular and eddy viscosity \\
\hline$\rho$ & density \\
\hline$\sigma$ & cavitation number \\
\hline$\tau$ & total stress tensor \\
\hline$\omega$ & specific dissipation \\
\hline()$_{L}$ & liquid value \\
\hline()$_{V}$ & vapour value \\
\hline()$^{v}$ & viscous \\
\hline()$^{t}$ & turbulent \\
\hline
\end{tabular}


$\overline{()} \quad$ Reynolds average for single-phase flow

$\overline{\overline{()}} \quad$ phase average for two-phase flow

() mass weighted average (Favre average)

() $)^{\prime} \quad$ fluctuations with respect to the Reynolds and phase averages

()$^{\prime \prime} \quad$ fluctuations with respect to the mass weighted average

\section{Introduction}

Cavitation is a significant engineering phenomenon that occurs in fluid machinery, fuel injectors, marine propellers, nozzles, underwater bodies, etc. In most cases, cavitation is an undesirable phenomenon, significantly degrading performance, resulting in reduced flow rates, lower pressure increases in pumps, load asymmetry, vibrations, noise and erosion.

Such flows are characterized by important variations of the local Mach number, compressibility effects on turbulence, and thermodynamic phase transition. For the simulation of these flows, the numerical method must accurately handle any Mach number. Moreover, the modelling of turbulence plays a major role in the correct simulation of unsteady behaviours. Sheet cavitation that appear on solid bodies are characterized by a closure region which always fluctuates, with the presence of a re-entrant jet. This jet is mainly composed of liquid which flows upstream along the solid surface. Reynolds-Averaged Navier-Stokes (RANS) models are frequently used to simulate such unsteady cavitating flows. One fundamental problem with this approach is that turbulence models are tuned by non-cavitating 
mean flow data. Moreover, the standard eddy-viscosity models based on the Boussinesq relation are known to over-produce eddy-viscosity, which reduces the development of the re-entrant jet and two-phase structure shedding [1]. The link to compressibility effects on turbulence is not clear. DNS of the supersonic boundary layer demonstrated a reduction in $k$ production as a consequence of compressibility $[2,3,4]$. In cavitating flows, the supersonic regime is reached in the mixture area because of the drastic diminution of the speed of sound. The detailed mechanisms of the interaction between turbulent flows and cavitation have not yet been clearly revealed, especially for phenomena occurring at small scales.

Different strategies have been investigated to limit or to correct standard turbulence models. An arbitrary modification was proposed by Reboud to reduce the turbulent viscosity [1], and has been used successfully by different authors $[5,6,7,8,9,10]$. The Shear Stress Tensor (SST) correction proposed by Menter $[11,12]$ to reduce the eddy-viscosity in case of positive pressure gradient and a variant based on realizability constraints [13] were tested for unsteady cavitating flows [14]. Other corrections are based on the modelling of compressibility effects of the vapour/liquid mixture in the turbulence model. Correction terms proposed by Wilcox [15] in the case of compressible flows were tested for unsteady periodic cavitating flows [6]. A sensitivity analysis of constants $C_{\varepsilon 1}$ and $C_{\varepsilon 2}$, which directly influence the production and dissipation of turbulence kinetic energy, was conducted for a $k-\varepsilon$ model and a cavitating hydrofoil case [16]. A $k-\ell$ model including a scale-adaptive term [17] was developped for cavitating flows [18]. This term allows the turbulence model to recognized the resolved scales in the flow and 
to adjust the eddy-viscosity as a consequence. Finally, a filter-based method was investigated [19] by which the sub-filter stresses are constructed directly using the filter size and the $k-\varepsilon$ turbulence closure.

The present work is part of research aimed at developing a numerical tool devoted to cavitating flows. A particular emphasis is placed on the investigation of closures for additional terms appearing on the mixture turbulence transport equations. These terms are due to the fact that the divergence of the phase velocity fluctuations is not null in two-phase flow, even if pure phases are assumed to be incompressible. Such terms (pressure-dilatation, mass flux, compressible dissipation) are similar to those involved in the compressible formulation of single-phase turbulence models. For high-speed aerodynamic flows, additional terms in the turbulent kinetic energy (TKE) equation have been modelled by Zeman [20, 21] and Sarkar [22, 23, 24]. It was showed that these dilatational terms act as sinks and, for sufficiently large turbulent Mach number, could lead to reduction of the turbulence levels. The compressible dissipation have been modelled by Sarkar and Wilcox [25, 15]. A synthesis of these different closures in the case of aerodynamic flows are presented in $[26,27,28]$.

An in-house finite-volume code solving the Reynolds-Averaged NavierStokes (RANS) compressible equations was developed with a homogeneous approach $[29,14,30]$. The cavitation phenomenon is modelled by a barotropic liquid-vapour mixture equation of state (EOS). The closures for additional terms proposed by Sarkar and Wilcox based on a turbulent Mach number 
are studied and compared. The influence of the cut-off turbulent Mach number is investigated for the Wilcox model. Various unsteady simulations are performed to compute two partial cavities for which the dynamic behaviour is different. Moreover, comparisons with results obtained with the Reboud compressibility correction, specially developped for cavitating flows are proposed. Our goals in this study are to investigate the effect of various compressibility corrections and turbulent closures for flows involving a cavitation sheet. This would help in gaining an insight into the range of predictions that we can expect with these models.

The paper is organized as follows: the theoretical formulation is summarized, including physical models and elements of the numerical methods. This is followed by sets of results on Venturi geometries and discussions.

\section{Governing equations and models}

The numerical simulations are carried out using an in-house code solving the one-fluid compressible RANS system. The homogeneous mixture approach is used to model two-phase flows. The phases are assumed to be sufficiently well mixed and the disperse particle size are sufficiently small thereby eliminating any significant relative motion. The phases are strongly coupled and moving at the same velocity. In addition, the phases are assumed to be in thermal and mechanical equilibrium: they share the same temperature $T$ and the same pressure $P$. The evolution of the two-phase flow can be described by the conservation laws that employ the representative flow properties as unknowns just as in a single-phase problem. 
To obtain the one-fluid equations, we use the phase average of a phase quantity $\phi_{k}$ defined by Ishii [31]:

$$
\overline{\overline{\phi_{k}}}=\frac{1}{T_{k}} \int \phi_{k} d \tau
$$

where $T_{k}$ is the time period of phase $k$. The mass-weighted average or Favre average is commonly used for compressible flows. In the following, an overtilde denotes a Favre average. Using this definition and starting from the instantaneous conservation equations, the equations for each phase can be expressed. The equation for mixture quantities are obtained by the summation of the separate equations of the phase quantities.

We introduce $\alpha_{k}$ the void fraction or the averaged fraction of presence of phase $k$. The density $\rho$, the center of mass velocity $u$ and the internal energy $e$ for the mixture are defined by:

$$
\begin{aligned}
\rho & =\sum_{k} \alpha_{k} \overline{\overline{\rho_{k}}} \\
\rho u_{i} & =\sum_{k} \alpha_{k} \overline{\overline{\rho_{k}}} \widetilde{u}_{k, i} \\
\rho e & =\sum_{k} \alpha_{k} \overline{\overline{\rho_{k}}} \widetilde{e}_{k}
\end{aligned}
$$

The mass gain per unit volume due to phase change (evaporation or condensation) is noted $\Gamma_{k}=\rho_{k}\left(u_{I}-u_{k}\right) \cdot n_{k} \delta_{I}$ where $\delta_{I}$ is a Dirac distribution having the different interfaces as a support, $u_{I}$ the interface velocity and $n_{k}$ the vector normal to the interface directed outward from phase $k$. 


\subsection{The mixture conservation laws}

The classical form for the homogeneous mixture balance equations are (see [31]):

$$
\begin{aligned}
\frac{\partial \rho}{\partial t}+\frac{\partial\left(\rho u_{i}\right)}{\partial x_{j}} & =0 \\
\frac{\partial}{\partial t}\left(\rho u_{i}\right)+\frac{\partial\left(\rho u_{i} u_{j}+P \delta_{i j}\right)}{\partial x_{j}} & =\frac{\partial\left(\tau_{i j}^{v}+\tau_{i j}^{t}\right)}{\partial x_{j}} \\
\frac{\partial}{\partial t}(\rho E)+\frac{\partial\left[(\rho E+P) u_{i}\right]}{\partial x_{j}} & =\frac{\partial\left[\left(\tau_{i j}^{v}+\tau_{i j}^{t}\right) u_{j}-Q_{i}^{v}-Q_{i}^{t}\right]}{\partial x_{j}}
\end{aligned}
$$

where $E$ is the mixture total energy. The mixture total stress tensor $\tau_{i j}$ (with viscous and turbulent contributions) is evaluated using the Stokes hypothesis, Newton's law and the Boussinesq assumption. The mixture total heat flux vector $Q_{i}$ is obtained from the Fourier law involving a turbulent thermal conductivity $\lambda_{t}$ with the constant Prandtl number hypothesis.

$$
\begin{aligned}
& \tau_{i j}=\tau_{i j}^{v}+\tau_{i j}^{t}=\left(\mu+\mu_{t}\right)\left[\left(\frac{\partial u_{i}}{\partial x_{j}}+\frac{\partial u_{j}}{\partial x_{i}}\right)-\frac{2}{3} \frac{\partial u_{l}}{\partial x_{l}} \delta_{i j}\right]+\frac{2}{3} \rho k \delta_{i j} \\
& Q_{i}=Q_{i}^{v}+Q_{i}^{t}=-\left(\lambda+\lambda_{t}\right) \frac{\partial T}{\partial x_{i}} \quad \text { with } \quad \lambda_{t}=\frac{\mu_{t} C_{p}}{P_{r t}}
\end{aligned}
$$

where $\mu_{t}$ is the mixture eddy viscosity, $k$ the mixture turbulent kinetic energy (TKE), $C_{p}$ the mixture thermal capacities and $P_{r t}$ the turbulent Prandtl number set to 1 .

In pure liquid, the viscosity is determined by an exponential law and, in pure vapour, the viscosity follows the Sutherland law. The mixture viscosity is defined as the arithmetic mean of the liquid and vapour viscosities (fluctuations of viscosity are neglected) [31]:

$$
\overline{\overline{\mu_{L}}}=\mu_{0_{L}} \exp (B / T)
$$




$$
\begin{aligned}
\overline{\overline{\mu_{V}}} & =\mu_{0_{V}} \sqrt{\frac{T}{293}} \frac{1+T_{S} / 293}{1+T_{S} / T} \\
\mu & =\sum_{k} \alpha_{k} \overline{\overline{\mu_{k}}}
\end{aligned}
$$

where $\mu_{0_{L}}, \mu_{0_{V}}, B$ and $T_{S}$ are constants. Subscripts $L$ and $V$ denotes liquid and vapour quantities.

The mixture thermal conductivity $\lambda$ is also defined as the arithmetic mean of the liquid and vapour values:

$$
\lambda=\sum_{k} \alpha_{k} \frac{\overline{\overline{\mu_{k}}} C_{p_{k}}}{P_{r_{k}}}
$$

\subsection{The cavitation model}

To close the system, an equation of state (EOS) is necessary to link the pressure to the thermodynamic variables. Pure phases follow the stiffened gas EOS. The barotropic law proposed by Delannoy [32] is considered for the mixture.

This law is characterized by its maximum slope $1 / c_{\text {baro }}^{2}$. The quantity $c_{\text {baro }}$ is an adjustable parameter of the model, which can be interpreted as the minimum speed of sound in the mixture.

When the pressure is between $P_{v a p}+\Delta P$ and $P_{v a p}-\Delta P$, the following relationship applies:

$$
P=P_{v a p}+\left(\frac{\overline{\overline{\rho_{L}}}-\overline{\overline{\rho_{V}}}}{2}\right) c_{b a r o}^{2} \operatorname{Arcsin}\left(1-2 \alpha_{V}\right)
$$

where $\Delta P$ represents the pressure range of the law and, for a void ratio value of 0.5 , the pressure is equal to the saturation pressure $P_{v a p}$. This law introduces a small non-equilibrium effect on the pressure. The cavitation 
phenomenon is assumed to be isothermal and the thermodynamic effect is neglected.

The hyperbolicity and convexity of the EOS have been demonstrated in [29]. The influence of $c_{\text {baro }}$ has been studied in previous works. In the present paper, the value of $c_{\text {baro }}$ is set to $0.472 \mathrm{~m} / \mathrm{s}$, corresponding to a pressure range of $\Delta P=175 \mathrm{~Pa}$.

\subsection{Turbulence modelling}

In the present study, two popular transport-equation turbulence models were used: the Wilcox $k-\omega$ model $(\mathrm{KO})[33,15]$ and the Jones-Launder $k-\varepsilon$ model (KE) [34].

In this section, we present both the turbulent transport-equation for single and two-phase flow to put in evidence the complexity of additional terms due to the mixture.

\subsubsection{Turbulence equations in high-speed aerodynamic flows}

\section{TKE equation}

The Favre-averaged turbulent kinetic energy $k$ is defined by $k=\frac{1}{2} \widetilde{u_{i}^{\prime \prime} u_{i}^{\prime \prime}}$. The turbulent kinetic energy equation writes in compressible turbulence (see $[27])$ :

$$
\frac{\partial \bar{\rho} k}{\partial t}+\frac{\partial \bar{\rho} \widetilde{u}_{l} k}{\partial x_{l}}=\bar{\rho} P_{k}+\bar{\rho} \Pi-\bar{\rho} \varepsilon+\bar{\rho} M+\bar{\rho} D
$$

with

$$
\begin{aligned}
\bar{\rho} P_{k} & =\frac{\tau_{i l} \frac{\partial \widetilde{u}_{i}}{\partial x_{l}}}{\bar{\rho}^{\prime}}=\frac{\partial u_{i}^{\prime \prime}}{\partial x_{i}}
\end{aligned}
$$




$$
\begin{aligned}
& \bar{\rho} \varepsilon=\overline{\sigma_{i l}^{\prime} \frac{\partial u_{i}^{\prime \prime}}{\partial x_{l}}} \\
& \bar{\rho} M=\frac{\overline{\rho^{\prime} u_{i}^{\prime \prime}}}{\bar{\rho}}\left(\frac{\partial \bar{P}}{\partial x_{i}}-\frac{\partial \overline{\sigma_{i l}}}{\partial x_{l}}\right) \\
& \bar{\rho} D=-\frac{\partial}{\partial x_{l}}\left[\bar{\rho} \widetilde{u_{i}^{\prime \prime} u_{i}^{\prime \prime} u_{l}^{\prime \prime}}+\overline{P^{\prime} u_{i}^{\prime \prime}} \delta_{i l}-\overline{\sigma_{i l}^{\prime} u_{i}^{\prime \prime}}\right]
\end{aligned}
$$

where $P_{k}$ is the production of TKE by the mean stain rate, $\varepsilon$ is the TKE dissipation rate, $D$ is the turbulent and viscous diffusions, $\Pi$ is the pressuredilatation correlation, $M$ is the turbulent mass flux. It is noteworthy that the terms $\Pi$ and $M$ are specifics to compressible turbulence.

The production term is closed following the Boussinesq assumption. The turbulent and viscous diffusions $D$ is closed using a gradient diffusion formulation:

$$
\bar{\rho} D=\left(\mu+\frac{\mu_{t}}{\sigma_{k}}\right) \frac{\partial k}{\partial x_{l}}
$$

If the viscosity fluctuations are neglected, the dissipation rate $\varepsilon$ can be approximated by:

$$
\bar{\rho} \varepsilon=2 \bar{\mu} \overline{s_{i k}^{\prime} s_{k i}^{\prime}}-\frac{2}{3} \bar{\mu} \overline{s_{k k}^{\prime} s_{l l}^{\prime}}
$$

after some rearrangements:

$$
\bar{\rho} \varepsilon=\underbrace{2 \bar{\mu} \overline{\omega_{i k}^{\prime} \omega_{i k}^{\prime}}}_{\bar{\rho} \varepsilon_{s}}+\underbrace{2 \bar{\mu} \frac{\partial}{\partial x_{k}}\left[\frac{\partial \overline{u_{k}^{\prime} u_{l}^{\prime}}}{\partial x_{l}}-2 \overline{u_{k}^{\prime} s_{l l}^{\prime}}\right]}_{\bar{\rho} \varepsilon_{i n h}}+\underbrace{\frac{4}{3} \bar{\mu} \overline{s_{k k}^{\prime} s_{l l}^{\prime}}}_{\bar{\rho} \varepsilon_{d}}
$$

with:

$$
s_{i j}^{\prime}=\frac{1}{2}\left(\frac{\partial u_{i}^{\prime}}{\partial x_{j}}+\frac{\partial u_{j}^{\prime}}{\partial x_{i}}\right) \quad \text { and } \quad w_{i j}^{\prime}=\left(\frac{\partial u_{i}^{\prime}}{\partial x_{j}}-\frac{\partial u_{j}^{\prime}}{\partial x_{i}}\right)
$$

$\varepsilon_{s}$ is the solenoidal dissipation, $\varepsilon_{i n h}$ is an inhomogeneous contribution often neglected and $\varepsilon_{d}$ is the dilatation dissipation rate. The solenoidal dissipation 
can be interpreted as an incompressible contribution whereas the dilatation dissipation is associated with compressible turbulence. In most of turbulent flow computations, only the solenoidal dissipation is taken into account.

The pressure-dilatation term $\Pi=\overline{P^{\prime} \frac{\partial u_{i}^{\prime \prime}}{\partial x_{i}}}$ has been modelled separately by Zeman [35] and Sarkar [24]. Zeman proposed a model based on a relaxation relation for the fluctuating pressure-variance associated with an acoustic time scale. These developments lead to the following relation:

$$
\bar{\rho} \Pi=\alpha\left(\frac{\overline{P^{\prime 2}}}{P_{e}^{2}}-1\right) \bar{\rho} \epsilon_{s} M_{t}+\beta \frac{\overline{P^{\prime 2}}}{\gamma \bar{P}} \widetilde{S}_{k k}
$$

where $P_{e}$ is the pressure-variance equilibrium value, $M_{t}=\frac{\sqrt{k}}{2 c}$ is the turbulent Mach number, $\alpha$ and $\beta$ are closure coefficients. In contrast, Sarkar applied a decomposition of the fluctuating pressure field $P^{\prime}$ into an incompressible part $P^{\prime I}$ and a compressible part $P^{\prime} C$. Using DNS results, he showed that only the incompressible part plays a role in compressible turbulence. Therefore, based on rapid distorsion theory, Sarkar proposed the following form for the term $\Pi$ :

$$
\bar{\rho} \Pi=-\alpha_{2} \bar{\rho} P_{k} M_{t}+\alpha_{3} \bar{\rho} \epsilon_{s} M_{t}^{2}
$$

with $\alpha_{2}=0,15$ and $\alpha_{3}=0,2$.

Many attempts have been tested to propose a model for the turbulent mass flux $\overline{\rho^{\prime} u_{i}^{\prime \prime}}$, which is an artifact of Favre-averaging. Some models based on a polytropic approach for the fluid [36] allowed to express the turbulent mass flux in function of the turbulent heat flux $\overline{T^{\prime} u_{i}^{\prime \prime}}$. Another approach, proposed by Jones [37] and taken up by Sarkar and Balakrishnan [38], modelled the 
turbulent mass flux by a gradient diffusion formulation:

$$
\overline{\rho^{\prime} u_{i}^{\prime \prime}}=-\frac{\mu_{t}}{\bar{\rho} \sigma_{p}} \frac{\partial \bar{\rho}}{\partial x_{i}}
$$

where $\sigma_{p}$ is a turbulent Schmidt number close to one. Including this model into the term $M$, it leads to:

$$
\bar{\rho} M=-\frac{\mu_{t}}{\bar{\rho}^{2} \sigma_{p}} \frac{\partial \bar{\rho}}{\partial x_{i}} \frac{\partial \bar{P}}{\partial x_{i}}
$$

TKE dissipation rate equation

As shown in equation (17), the turbulent dissipation is split in three terms and only the solenoidal dissipation is solved in turbulent flow computations since turbulence model are built for incompressible turbulence. Therefore, only a transport equation for the solenoidal dissipation $\varepsilon_{s}$ is available in the literature [27]. Due to the complex form of the exact equation, only a simplified dissipation equation is built by imitation with the turbulent kinetic energy equation.

Nevertheless, to take into account the dilatation dissipation $\varepsilon_{d}$ in the turbulent kinetic energy equation, Zeman [20] and Sarkar [39] proposed a model for $\varepsilon_{d}$. Wilcox [40] also proposed a model adapted to his $k-\omega$ turbulence model. These models consist in expressing the dilatation dissipation $\varepsilon_{d}$ as a function of the solenoidal dissipation $\varepsilon_{s}$ and the turbulent Mach number $M_{t}$ :

$$
\varepsilon_{d}=\varepsilon_{s} F\left(M_{t}\right)
$$

with:

$$
F\left(M_{t}\right)= \begin{cases}\alpha_{1} M_{t}^{2} & \text { Sarkar } \\ \frac{3}{4}\left[1-e^{-\frac{1}{2}(\gamma+1)\left[\left(M_{t}-M_{t 0}\right) / 0.6\right]^{2}}\right] H\left(M_{t}-M_{t 0}\right) & \text { Zeman } \\ \xi^{*}\left(M_{t}^{2}-M_{t 0}^{2}\right) H\left(M_{t}-M_{t 0}\right) & \text { Wilcox }\end{cases}
$$


where $H$ is the Heavyside function, $M_{t 0}$ a cut-off turbulent Mach number set to 0.1 for the Zeman model and to 0.25 for the Wilcox model, constants $\xi^{*}=1.5$ and $\alpha_{1}=0.5$.

\subsubsection{Mixture turbulence equations for two-phase flows}

TKE equation for the mixture

Different authors have studied the turbulent kinetic energy equation for a gas-liquid flow. The mixture equation can be obtained by the summation of the separate equations of the phase turbulent quantities with homogeneous mixture assumptions $[41,42,43,44]$. Another approach consists in subtracting from the averaged kinetic energy equation the kinetic energy equation of the averaged field.

The TKE equation for the mixture is:

$$
\frac{\partial \rho k}{\partial t}+\frac{\partial \rho k u_{l}}{\partial x_{l}}=\rho P_{k}+\rho \Pi-\rho \epsilon+\rho M+\rho D+\Gamma
$$

where $k=\sum_{k} \alpha_{k} \frac{\widehat{u_{k, i}^{\prime \prime} u_{k, i}^{\prime \prime}}}{2}, P_{k}$ is the mixture production term, $\Pi$ is the mixture pressure-dilatation term, $\varepsilon$ is the mixture TKE dissipation rate which can be split into a solenoidal part $\varepsilon_{s}$ and a dilatational part $\varepsilon_{d}, M$ is the mixture turbulent mass flux, $D$ is the mixture turbulent and viscous diffusions. The last term $\Gamma$ is specific to multiphase flows. It involves the mass transfer due to phase change and is usually set to zero.

In multiphase flow, even if pure phases are assumed to be incompressible, the divergence of the fluctuating phase velocity is not zero, caused by the 
presence of interfaces. Indeed, with this assumption, the divergence of the fluctuating phase velocity is:

$$
\frac{\partial u_{k, i}^{\prime \prime}}{\partial x_{i}}=-\frac{\partial \widetilde{u_{k, i}}}{\partial x_{i}}=\frac{1}{\alpha_{k}} u_{k}^{\prime \prime} \cdot n_{k} \delta_{I}
$$

Therefore, the dilatational dissipation rate $\varepsilon_{d}=\frac{4}{3} \sum_{k} \alpha_{k} \overline{\overline{\mu_{k}}} \overline{\overline{s_{k, j j}^{\prime} s_{k, l l}^{\prime}}}$ is not zero. And the pressure-dilation term $\Pi=\sum_{k} \alpha_{k} \overline{\overline{P_{k}^{\prime} \frac{\partial u_{k, i}^{\prime \prime}}{\partial x_{i}}}}$ is instantaneously present but null in mean as $\overline{\overline{P_{k}^{\prime}}}=0$.

The modelling of these additional terms is very complex to realize in turbulent cavitating flows. This fact is linked with the experimental difficulties that arise when measurements must be performed in bubbly mixtures. Moreover, direct simulations (DNS) of two-phase flows involving a phase transition remains a challenging problem. For these reasons, we decide to take into account these terms by using the closure relations developed for aerodynamic high-speed flows.

\section{TKE dissipation rate equation for the mixture}

Kataoka and Serizawa [43] derived a transport equation for the mixture turbulent dissipation rate assuming that both phases were considered as incompressible. This equation put in evidence that the turbulent dissipation is affected by interfacial transport terms. Nevertheless, no theoretical or experimental results regarding these terms are available in case of two-phase flows with phase change. Therefore, we decide to use the same equation for $\varepsilon$ than in single-phase flows. 


\subsubsection{A $k-\varepsilon$ model in compressible form}

We include the compressible terms $\Pi, M$ and $\varepsilon_{d}$ into the Jones- Launder $k-\varepsilon$ model:

$$
\begin{aligned}
\frac{\partial \rho k}{\partial t}+\frac{\partial}{\partial x_{j}}\left[\rho k u_{j}-\left(\mu+\frac{\mu_{t}}{\sigma_{k}}\right) \frac{\partial k}{\partial x_{j}}\right] & =P_{k}-\rho \varepsilon_{s}+\rho \varepsilon_{d}+\rho \Pi+\rho M \\
\frac{\partial \rho \varepsilon_{s}}{\partial t}+\frac{\partial}{\partial x_{j}}\left[\rho \varepsilon_{s} u_{j}-\left(\mu+\frac{\mu_{t}}{\sigma_{\varepsilon}}\right) \frac{\partial \varepsilon_{s}}{\partial x_{j}}\right] & =c_{\varepsilon 1} \frac{\varepsilon_{s}}{k} P_{k}-\rho c_{\varepsilon 2} f_{2} \frac{\varepsilon_{s}^{2}}{k}+E
\end{aligned}
$$

The low Reynolds number term $E$, the damping functions $f_{\mu}$ and $f_{2}$ and all constants follow the Jones-Launder formulation [34].

The additional terms $\Pi$ and $\varepsilon_{d}$ are modelled according to Sarkar propositions:

$$
\begin{aligned}
\rho \Pi & =-\alpha_{2} \rho P_{k} M_{t}+\alpha_{3} \rho \varepsilon_{s} M_{t}^{2} \\
\varepsilon_{d} & =\alpha_{1} \varepsilon_{s} M_{t}^{2}
\end{aligned}
$$

where the turbulent Mach number $M_{t}$ is defined with the mixture speed of sound $c$.

The turbulent mass flux $M$ is closed with the Jones formulation:

$$
\rho M=-\frac{\mu_{t}}{\rho^{2} \sigma_{p}} \frac{\partial \rho}{\partial x_{i}} \frac{\partial P}{\partial x_{i}}
$$

with a turbulent Schmidt number $\sigma_{p}$ set to 1.

\subsubsection{A $k-\omega$ model in compressible form}

Wilcox [15] proposed a compressible version of his $k-\omega$ model:

$$
\begin{aligned}
\frac{\partial \rho k}{\partial t}+\frac{\partial}{\partial x_{j}}\left[\rho k u_{j}-\left(\mu+\sigma^{*} \mu_{t}\right) \frac{\partial k}{\partial x_{j}}\right] & =P_{k}-\beta_{c}^{*} \rho k \omega \\
\frac{\partial \rho \omega}{\partial t}+\frac{\partial}{\partial x_{j}}\left[\rho \omega u_{j}-\left(\mu+\sigma \mu_{t}\right) \frac{\partial \omega}{\partial x_{j}}\right] & =\alpha \frac{\omega}{k} P_{k}-\beta_{c} \rho \omega^{2}
\end{aligned}
$$


with $\mu_{t}=\rho \frac{k}{\omega}$. The compressibility effects on turbulence is accounted through the coefficients $\beta_{c}^{*}$ and $\beta_{c}$ functions of the turbulent Mach number:

$$
\begin{aligned}
\beta_{c}^{*} & =\beta^{*}\left(1+\xi^{*} F\left(M_{t}\right)\right) \\
\beta_{c} & =\beta-\beta^{*} \xi^{*} F\left(M_{t}\right) \\
F\left(M_{t}\right) & =\left(M_{t}^{2}-M_{t 0}^{2}\right) H\left(M_{t}-M_{t 0}\right)
\end{aligned}
$$

with $M_{t 0}=0.25$ and $\xi^{*}=1.5$.

\subsubsection{Compressibility correction for cavitating flows}

To account for compressibility effects in cavitating flows, Reboud [1] proposed an arbitrary limiter by introducing a function $f(\rho)$ in the computation of the turbulent viscosity for the $k-\varepsilon$ model:

$$
\mu_{t}=f(\rho) C_{\mu} \frac{k^{2}}{\varepsilon} \quad \text { with } f(\rho)=\rho_{V}+(1-\alpha)^{n}\left(\rho_{L}-\rho_{V}\right)
$$

where $n$ is a parameter usually set to 10 . This function can be used for other two-equation models.

\subsubsection{Wall modelling}

For the modelling of flow close to the wall, a two-layer wall law approach is used. We assume that wall functions are similar in two-phase and singlephase flows. For unsteady flows, the existence of a wall law is assumed to be valid at each instant. More details concerning the wall law approach are given in [29].

\section{Numerical methods}

The numerical simulations are carried out using an implicit CFD code solving the RANS/turbulent systems for multi-domain structured meshes. 
This solver is based on a cell-centered finite-volume discretization.

For low Mach number applications, a well-known problem concerns the stiffness on the solution convergence when the Mach number becomes low. In this situation, the dominance of convection terms renders the system stiff and compressible solvers converge slowly. To overcome this difficulty, a preconditioned method is necessary. The physical acoustic waves are replaced by pseudo-acoustic modes that are much closer to the advective velocity, reducing the stiffness and enhancing the convergence. In this work, we use an inviscid preconditioning method based on the modification of the derivative term by a pre-multiplication with a suitable preconditioning matrix $P_{c}$ [45]. The detail of the formulation is given in [29].

The system in integral form is written for a computational cell of volume $\Omega$ limited by a surface $\Sigma$, with an outer normal $n$. It can be expressed as:

$$
P_{c}^{-1} \frac{d}{d t} \int_{\Omega} w d \Omega+\oint_{\Sigma} F_{c} \cdot n d \Sigma-\oint_{\Sigma} F_{d} \cdot n d \Sigma=\int_{\Omega} S d \Omega
$$

where $w$ denotes the conservative variables, $F_{c}$ and $F_{d}$ the convective and dissipative flux densities, and $S$ the source terms, which concern only the turbulence transport equations.

\subsection{Spatial discretization}

Using the finite-volume technique for space discretization, a semi-discrete form of equation (37) is written. For the mean flow, the convective flux density vector on a cell face is computed with the Jameson scheme [46] in which the dispersive error is cancelled. The artificial viscosity includes a 
second-order dissipation term $D_{2}$ and a fourth-order dissipation term $D_{4}$, which involve two tunable parameters $k^{(2)}$ and $k^{(4)}$.

The viscous terms are discretized by a second-order space-centered scheme. For the turbulence transport equations, the upwind Roe scheme [47] is used to obtain a more robust method. The second-order accuracy is obtained by introducing a flux-limited dissipation [48].

\subsection{Temporal discretization}

Time integration is achieved using a low-cost implicit method [49]. The implicit method consists in solving, at each time step, a system of equations arising from the linearization of a fully implicit scheme. The main advantage of this method is that the storage of the Jacobian matrix is completely eliminated, which leads to a low-storage algorithm. More details are given in $[29]$.

For the turbulence transport equations, the diffusive flux Jacobian matrix is replaced by its spectral radius. The source term needs special treatment [50]. Only the negative part of the source term Jacobian matrix is considered and replaced by its spectral radius. The system obtained is solved with a linealternated Jacobi relaxation algorithm.

For unsteady computations, the dual time stepping method, proposed by Jameson [51], was used to solve the lack of numerical efficiency of the global time stepping approach. The derivative with respect to the physical time is discretized by a second-order formula. Making the scheme implicit with respect to the dual time provides fast convergence to the time-accurate solution. Between each time step, the solution is advanced in a dual time, and 
acceleration strategies developed for steady problems can be used to speed up the convergence in fictitious time. With respect to physical time, the derivative was initialized with a first-order formula.

\subsection{Inlet and outlet boundary conditions}

The numerical treatment of boundary conditions is based on the use of the preconditioned characteristic relationships [29]. We assume that inlet and outlet areas are in a pure liquid region; no cavitation appears in these boundaries.

\section{The test cases: partial cavities on Venturi geometries}

\subsection{Experimental data}

Experiments have been performed in a closed loop at CREMHyg (Centre d'Essais de Machines Hydrauliques de Grenoble) and the test section is 520 $\mathrm{mm}$ long, $44 \mathrm{~mm}$ wide and $50 \mathrm{~mm}$ high in the inlet. Two flow configurations have been tested corresponding to flat upper and side walls, whereas the height of the lower wall varied in order to form a convergent-divergent nozzle. In the present considered experiments, case 1 was constituted by an angle of the divergent part of $4^{\circ}$. Case 2 corresponds to a convergent part of about $18^{\circ}$ and the divergent part was $8^{\circ}$. The pressure $P_{\text {inlet }}$ was lowered until the desired cavitation number allowing two specific behaviours of the sheet cavity. The cavitation number in the inlet section is defined as: $\sigma_{\text {inlet }}=\frac{P_{\text {inlet }}-P_{\text {vap }}}{0.5 \rho U_{\text {inlet }}^{2}}$, where $P_{\text {vap }}$ is the vapour pressure at $20^{\circ} \mathrm{C}$ and $P_{\text {inlet }}, U_{\text {inlet }}$ are the pressure and velocity respectively at the reference section upstream of the Venturi. The geometrical data and flow configurations 
are given in Table (1).

With these previous parameters and according to experimental observations $[52,7]$, cavitation sheets developed from the Venturi throat. The obtained cavity length is ranging from $70 \mathrm{~mm}$ to $85 \mathrm{~mm}$ for case 1 and having a relatively stable aspect (see Fig. 2). The attached cavity length corresponding to the end of the re-entrant jet is around $30 \mathrm{~mm}$. For this case, no periodic cycles with large shedding were observed.

For case 2, a typical self-oscillation behaviour was observed with quasiperiodic vapour clouds shedding and the maximum cavity length (before the break-off of the cavity) was $45 \pm 5 \mathrm{~mm}$. Figure 2 shows an instantaneous photograph of the cavity with a large structure shedding. The cloud shedding frequency was about $45 \mathrm{~Hz}$ leading to the classical Strouhal number based on the cavity length of about 0.3 . The divergent part was equipped with eight probe holes to take various measurements such as the instantaneous pressure, local void ratio and velocity in the sheet cavity. Specific probe locations are presented in Figure 1 for the two tested cases.

Pressure measurements were carried out using a piezoelectric sensor DRUCK model with a maximum range of $70000 \mathrm{~Pa}$ and absolute precision of \pm 19 $\mathrm{Pa}$. The mean and fluctuating values of the pressure at the wall have been measured upstream to characterize the cavitation number and at any stations in the divergent area. Fast Fourier transformation (FFT) analysis of pressure signal with a sampling rate of $50 \mathrm{kHz}$ and 6 millions of samples has been performed to estimate the shedding frequency. For the two-phase 
flow measurements, double optical probe was used to evaluate void ratio and velocity fields. It was constituted by two sapphire optical fibers of $80 \mathrm{~mm}$ diameter with frayed ends and inter-tips distance of about $1.05 \mathrm{~mm} \pm 0.02$. The relative uncertainty on the void ratio measurement was estimated at roughly $15 \%[52]$.

For these geometries, we do not have access to measurements of turbulent quantities in the two phase-flow region. This lack of information makes turbulence models validation difficult.

\subsection{Numerical data}

Both grids are a H-type topology. A special contraction of the mesh is applied in the main flow direction just after the throat to better simulate the two-phase flow area (Fig. 3).

For the non cavitating regime, computations are started from an uniform flow-field using a local time step. For the unsteady cavitating regime, computations are performed with the dual time stepping method and are started from the non cavitating numerical solution. The numerical parameters are given in Table (2).

All numerical values are obtained by a time-averaged treatment. For case 2, a direct Fourier transformation (DFT) of the vapour volume signal was performed to evaluate the frequency. 


\section{Results obtained with the $k-\varepsilon$ model}

The standard model without corrections provided quasi steady solutions in poor agreement with the experimental data. The add of the Reboud correction allows to largely improve results and make the model able to simulate an unsteady sheet with a re-entrant jet. Results obtained on both geometries with the $k-\varepsilon$ model coupled with the Reboud limiter have been presented in $[14]$.

The $k-\varepsilon$ model including all additional terms presented previously involves 4 constants: $\alpha_{1}, \alpha_{2}, \alpha_{3}$ and $\sigma_{p}$. Unfortunately, it is not possible to calibrate these constants for cavitating flows due to the lack of turbulent data. We decided to conserve the Sarkar choice and to fix the turbulent Schmidt number $\sigma_{p}$ to 1 .

Secondly, in order to separately evaluate the influence of the additional terms (the mass flux term $M$, the pressure-dilatation term $\Pi$ and the dilatational dissipation $\varepsilon_{d}$ ), we performed different simulations of the case 1 by varying the constants value. The goal was to obtain a cavity sheet characteristic close to the experimental data. With the new constants values, we computed the cavity of the case 2 .

In the following, we propose first a qualitative description of the simulated cavities with plotting of the contours of the density gradient modulus (Schlieren-like visualizations). Secondly, local comparisons with velocity and void ratio profiles are presented in paragraph 5.2. All unsteady calculations performed on both geometries are summarized in Table (3). The time of 
simulation is around 2 seconds.

\subsection{Qualitative description of cavities}

\subsubsection{Compressible model with all additional terms}

The partial cavity of the case 1 was computed with the $k-\varepsilon$ model including all additional terms following the formulation proposed by Sarkar (KE compressible). A qualitative view of the cavity sheet is plotted in Figure 4 with the contours of the density gradient modulus. We obtained a very small attached cavity to the throat up to the abscissa $x=0.01 \mathrm{~m}$ instead of $0.03 \mathrm{~m}$ in the experimental data. Downstream, small clouds of cavitation are convected up to $x=0.15 \mathrm{~m}$. The time-averaged cavity length is around $0.12 \mathrm{~m}$ instead of $0.08 \mathrm{~m}$ in the experiment.

The partial cavity of the case 2 was computed with the KE compressible model. The predicted frequency is around $19 \mathrm{~Hz}$ instead of $45 \mathrm{~Hz}$ in the experimental data. The contours of the density gradient modulus are illustrated in Figure 5 during one period. We can observe the very large structure shedding up to $\mathrm{x}=0.15 \mathrm{~m}$.

\subsubsection{Mass flux term}

The partial cavity of the case 1 was computed with the $k-\varepsilon$ model only including the mass flux term $M$ in the mixture TKE equation (KE mass). The turbulent Schmidt number $\sigma_{p}$ was set to one. Results obtained are quite similar to those obtained with the standard model: the attached cavity length is around $0.065 \mathrm{~m}$ (instead of $0.03 \mathrm{~m}$ in the experiment) and the reentrant jet is largely under-estimated. The influence of the Schmidt number 
value was tested by decreasing the $\sigma_{p}$ value up to 0.001 ; only a quasi steady behaviour of the sheet cavity was computed. The contours of the density gradient modulus are illustrated in Figure 6, computed with $\sigma_{p}=1$ (left) and $\sigma_{p}=0.001$ (right). Both simulations exhibit a large attached cavity and a small recirculation on the closure part. Results are summarized in Table (4).

To conclude, with the closure of the mass flux term following the Jones formulation, this term can be neglected.

\subsubsection{Pressure-dilatation term}

The partial cavity of the case 1 was computed with the $k-\varepsilon$ model only including the pressure-dilatation term $\Pi$ in the mixture TKE equation (KE pd). The sign of $\Pi$ can vary, it can act as a production or destruction term:

$$
\rho \Pi=-\alpha_{2} \rho P_{k} M_{t}+\alpha_{3} \rho \varepsilon_{s} M_{t}^{2} \quad \text { with } \alpha_{2}=0.15 ; \alpha_{3}=0.2
$$

With the aerodynamic cases used by Sarkar to calibrate the constants, the turbulent Mach number $M_{t}$ is smaller than 1, and so $M_{t}^{2}$ is small. In our cavitating cases, due to the drastic reduction of the speed of sound in the mixture (less than $1 \mathrm{~m} / \mathrm{s}$ ), values of $M_{t}$ are upper to 1 . As a consequence, the term acts as a production term. It was not possible to obtain a convergence result with the original formulation.

We decided to conserve $\alpha_{2}$ and to reduce the constant $\alpha_{3}$ from 0.05 to 0.001 so as to obtain a negative value for $\Pi$. Three types of sheet cavity were simulated:

- A quasi steady cavity characterized by an extended attached cavity (up 
to $0.065 \mathrm{~m}$ ) and a very small recirculating area at the closure part. This situation is similar to that obtained with standard turbulence models and with the KE mass model (see Figure 6). Such cavity was predicted with $\alpha_{3}=0.05$.

- A cavity sheet with large break off and clouds shedding. It is characterized by a small attached cavity (around $0.02 \mathrm{~m}$ ) and a large structure shedding downstream, up to the abscissa $x=0.2 m$. Such cavity was predicted with $\alpha_{3}=0.001$. The cavity description is illustrated in Figure 7 where is plotted the contour of the instantaneous density gradient modulus. The model provides cavitation cloud shedding not observed in the experiment.

- An intermediate situation in better agreement with the experimental visualization. The sheet cavity is characterized by a attached cavity length varying between 0.025 and $0.04 \mathrm{~m}$, and the cavitation cloud shedding is moderately extended. Such cavity was predicted with $\alpha_{3}=$ 0.025. The contours of the density gradient modulus are presented in Figure 8 at three different instants. A stable attached cavity is signaled by a strong density gradient from the throat up to the abscissa $x=0.025 \mathrm{~m}$. This cavity is followed by a time-fluctuating two-phase area with clouds shedding and a shear layer is clearly exhibited.

Results are summarized in Table (4). Smaller is the value of $\alpha_{3}$, more extended and intense is the re-entrant jet. Indeed, the destruction of TKE due to the pressure-dilatation term increases, the turbulent viscosity is reduced allowing the development of the re-entrant jet. 
The partial cavity of the case 2 was computed with the KE pd model with $\alpha_{3}=0.025$. The self-oscillating behaviour of the cavity is simulated with a frequency around $21 \mathrm{~Hz}$ (see Table 3) instead of $45 \mathrm{~Hz}$ in the experiment. An overview of the sheet dynamic during one period is illustrated in Figure 9 with the contours of density gradients. A very small attached cavity is exhibited on the throat and two-phase structures are convected in the divergent up to the abscissa $x=0.05 \mathrm{~m}$, in correct agreement with the experimental visualizations.

\subsubsection{Extra-dissipation term}

The partial cavity of the case 1 was computed with the $k-\varepsilon$ model only including the dilatational dissipation term $\varepsilon_{d}$ in the mixture TKE equation (KE epsd). The closure relation proposed by Sarkar involves a constant $\alpha_{1}$ set to 0.5. Different values were tested in order to evaluate its influence on the sheet cavity dynamics, from 0.5 to 0.01 .

We simulated the three types of sheet cavity as described previously with the pressure-dilatation term. The quasi steady sheet cavity was obtained with the smallest tested value $\alpha_{1}=0.01$. A cavity with large clouds shedding was predicted with the original value $\alpha_{1}=0.5$, in which the time-averaged cavity length was around $0.22 \mathrm{~m}$ instead of $0.08 \mathrm{~m}$ in the experiment. The intermediate case was simulated with an intermediate value $\alpha_{1}=0.05$. For this cavity, the contours of the density gradient modulus are illustrated in Figure 10 at three different instants. We can see the attached cavity from the throat up to the abscissa varying between $x=0.035 \mathrm{~m}$ and $x=0.045 \mathrm{~m}$. Behind, cavitation clouds are exhibited up to $x=0.12 \mathrm{~m}$. Results are summarized in 
Table (4). Conclusions are similar to those given with the pressure-dilatation term. Higher is the extra-dissipation term, more reduced is the turbulent viscosity and more extended is the re-entrant jet.

The partial cavity of the case 2 was computed with the KE epsd model with $\alpha_{1}=0.05$. Based on a DFT of the vapour volume, the computed frequency is around $20 \mathrm{~Hz}$ instead of $45 \mathrm{~Hz}$ in the experimental data. The contours of the density gradient modulus are illustrated in Figure 11 during one period. As observed with the KE pd model, a small attached cavity is always fixed on the throat. Downstream, the two-phase structure shedding is not enough extended.

\subsection{Local comparisons of compressible corrections}

Four models are compared: the $k-\varepsilon$ model with all additional terms proposed by Sarkar (KE compressible), the $k-\varepsilon$ model including the pressuredilatation term with $\alpha_{3}=0.025$ (KE pd), the $k-\varepsilon$ model including the dilatational dissipation term with $\alpha_{1}=0.05$ (KE epsd) and the $k-\varepsilon$ model coupled with the Reboud limiter (KE Reboud).

\subsubsection{Case 1}

With models including compressible terms, the cavitation parameter in the inlet section $\sigma_{\text {inlet }}$ is overestimated (around 0.63) whereas the value provided by the KE Reboud model is close to the experimental value (see Table (3)).

Figure 12 shows the longitudinal velocity profiles (on the left) and void 
ratio profiles (on the right) for the experiments and all computations. From station 3 to 5 , experimental observation indicates a recirculating behaviour with a re-entrant jet extending roughly through half the sheet thickness. We clearly observed on the velocity profiles that the re-entrant jet predicted by the KE compressible model is too extended. Indeed, at station 2 where no recirculation was measured, the model simulated a large separation area. As a consequence, the void ratio values at station 2 and 3 are largely underestimated. With the KE epsd model, at station 2, the velocity profile is near to separate and void ratio values are smaller than the experimental data. From station 3 to 5, all models predicted a re-entrant jet, differences appear on the thickness and the intensity of the separation. The profiles computed with the KE Reboud model are in good agreement with the experimental data.

The dimensionless time-averaged wall pressure distribution $\frac{P-P_{v a p}}{P_{\text {vap }}}$ is plotted in Figure 13 versus the distance $x-x_{\text {inlet }}$ for the experiments and all computations. The first five data are located inside the cavity (where the void ratio and velocity profiles are measured).

The KE Reboud model provided a pressure distribution in correct agreement with experimental measurements, the re-compression area downstream the cavity being a little under-estimated. With all other compressible KE models, the re-compression area is very badly predicted.

The Root Mean Square (RMS) wall pressure fluctuations are plotted in Figure 14 versus the distance $x-x_{\text {inlet }}$. The pressure fluctuation is divided 
by the time-averaged pressure Pav. Experimental data indicate an augmentation of pressure fluctuations at the end of the sheet cavity, with a peak located at the fifth station. The Reboud model yield a pressure fluctuation profile close to the experimental profile except for the pressure peak, located between the fourth and the fifth station and largely overestimated (factor 5). The pressure fluctuations simulated by the compressible model are very remote from the experimental data. The sheet cavity dynamics obtained with this model is in very poor agrement with the experimental data, as observed with the density gradient modulus. With the KE epsd and KE pd models, the pressure fluctuations show a peak between the fifth and the sixth station and largely overestimated (factor 3 for the KE epsd model and factor 2 for the KE pd model). Downstream, values remain at a too high level.

\subsubsection{Case 2}

Firstly, all models captured a periodic self-oscillating cavity. With all KE models including compressible terms, it was impossible to obtain a frequency close to the experimental value: around $20 \mathrm{~Hz}$ instead of $45 \mathrm{~Hz}$ in the experiment. On the contrary, the KE Reboud model simulated a quasi cyclic phenomenon with a frequency close to $45 \mathrm{~Hz}$.

Figure 15, on the left, shows the evolution of the longitudinal velocity profiles for the experiments and all computations, at stations 1 and 2. All models provided similar results with a good prediction of the re-entrant jet phenomenon.

Figure 15, on the right, illustrates experimental and numerical results concerning the void ratio profiles, from station 1 to 3 . Experimental values are 
weak, even at station 1. For this station, the KE Reboud model largely overpredicted the void ratio (up to a factor of 4), whereas the profile simulated by all other compressible models are close to the experimental data. At station 2, the void ratio is still over-predicted by the KE Reboud model. For the last station, the KE Reboud model under-estimated the cavity length and provided a quasi liquid profile. On the other hand, the compressible KE model captured large clouds shedding and void ratio values are too important (factor 8). Results obtained with the KE pd and KE epsd models are in correct agreement with the experimental data.

The dimensionless time-averaged wall pressure distribution $\frac{P-P_{v a p}}{P_{v a p}}$ is plotted in Figure 16 versus the distance $x-x_{\text {inlet }}$. The re-compression area is not well simulated by all models. The better result is given by the KE Reboud model.

\subsection{Partial conclusions}

These studies based on the Jones-Launder $k-\varepsilon$ model taking into account compressibility effects on turbulence lead to different conclusions:

- The mass flux term modelled by a gradient model can be neglected.

- The original model proposed by Sarkar is not adapted for cavitating flows. A recalibration of the model is necessary.

- Tests based on additional compressible terms as pressure-dilatation and dilatational dissipation show an improvement in comparison with the standard model. Yet, it was not possible to capture the frequency of the self-oscillating cavity. 
- The Reboud limiter, in spite of both its simplicity and empiricism, make the model able to predict unsteady behaviour in correct agreement and to compute the frequency of the periodic phenomenon.

\section{Results obtained with the Wilcox $k-\omega$ model}

As observed with the $k-\varepsilon$ turbulence model, the standard Wilcox model provided quasi steady solutions in poor agreement with the experimental data. We performed various simulations on both partial cavities including the compressible corrections proposed by Wilcox and the limiter proposed by Reboud. The unsteady calculations performed on both geometries are summarized in Table (5). The time of simulation is around 2 seconds.

\subsection{Influence of the cut-off turbulent Mach number}

The compressible corrections proposed by Wilcox (KO compressible) introduce a function $F\left(M_{t}\right)$, which involves a cut-off turbulent Mach number $M_{t_{0}}$ set to 0.25 . This function was calibrated from supersonic aerodynamic data where the turbulent Mach number $M_{t}$ varies between 0.1 to 0.7 . In cavitating flows, the drastic reduction of the speed of sound in the liquidvapor mixture leads to the existence of supersonic areas. In these regions, the turbulent Mach number $M_{t}$ can reach large values up to 3 . The complete recalibration of the compressible corrections for cavitating flows can not be realized due to the lack of experimental data. Only a study of the sensitivity to the cut-off value is proposed. 


\subsubsection{Case 1}

We tested different values of $M_{t_{0}}$ from the original value 0.25 to 2.5 . A qualitative description of the cavity is presented in Figure 17 through the contours of the instantaneous density gradients. On the left, with $M_{t_{0}}=0.25$, we can observe a very small attached cavity to the throat and the clouds cavitation shedding up to the abscissa $x=0.12 \mathrm{~m}$. This situation is in poor agreement with the experimental visualizations. On the right, with $M_{t_{0}}=2.5$, the cavity simulated is in better agreement with the experiments: the attached cavity is more extended with a small clouds shedding. With the intermediate values tested, $M_{t_{0}}=0.8$ and $M_{t_{0}}=1.5$, the attached cavity grows and the clouds shedding decreases.

To evaluate the influence of the value of $M_{t_{0}}$, we extracted from computations both the turbulent Mach number and density gradient modulus versus the abscissa $x$, at constant mesh line $j=12$. Five computations are compared: the Wilcox model without any compressible corrections and the Wilcox compressible model with $M_{t_{0}}=0.25,0.8,1.5$ and 2.5. Figure 18 plots the instantaneous turbulent Mach number (on the left) and density gradient modulus (on the right) for the five computations. With the standard $k-\omega$ model, we clearly observed the quasi steady behaviour. Large values of $M_{t}$ are reached in the attached cavity (around 2) and a peak appears at the closure part of the sheet. For all compressible models, the maximum value of $M_{t}$ depends on the cut-off value $M_{t_{0}}$. Larger is the cut-off Mach number, larger are the turbulent Mach number values. Indeed, a low cut-off Mach number leads to a large decrease of the turbulent kinetic energy due to large 
values of the $\beta^{*}$ and $\beta_{c}^{*}$ coefficients. As the speed of sound in the mixture is similar for all the computations, the turbulent Mach number decreases. That is also the case for the density gradient values. Near the throat, at the abscissa $x=0.02 \mathrm{~m}$, the density gradient values increase when the cut-off Mach number increases. For small and thin attached cavities (small value of $\left.M_{t_{0}}\right)$, the density gradient is weak at this ordinate $(j=12$ corresponds approximatively to the ordinate $y=0.009 \mathrm{~m}$ near the throat). When the attached cavity grows (large values of $M_{t_{0}}$ ), the cavity thickness increases and the density gradient reaches its maximum value. On the contrary, the maximum values of the density gradient are reached in the clouds cavitation for the lowest values of $M_{t_{0}}$. Moreover, a correlation between a peak of the gradient density and the turbulent Mach number is observed. This is explained by the low value of the speed of sound in the mixture and not by an increase of the turbulent intensity in the mixture.

Figure 19 shows the evolution of the longitudinal velocity (left) and the void ratio (right) for the experiments and the computations. At station 2, we clearly observed the negative values on the velocity profiles for models with $M_{t_{0}}=0.25$ to 1.5 . As a consequence the void ratio values are largely underestimated. Only the Wilcox model with the largest value $M_{t_{0}}=2.5$ provides profiles in correct agreement with the experimental data. Yet, at station 3, the void ratio values are under-predicted, similarly to those obtained with other KW models.

At stations 3 and 4, all models provided similar velocity profiles. The void ratio values are under-estimated by all models at station 4 , especially with 
$M_{t_{0}}=2.5$. At station 5, results are in good agreement with the experimental data, except with $M_{t_{0}}=2.5$ where the cavity length is not enough extended.

The dimensionless time-averaged wall pressure distribution $\frac{P-P_{v a p}}{P_{v a p}}$ is presented in Figure 20 versus the distance $x-x_{\text {inlet }}$. Results provided by the model with $M_{t_{0}}=1.5$ and $M_{t_{0}}=2.5$ are similar and in good agreement with the experimental data. With the other model, the attached cavity is not enough developped and the time-averaged pressure is higher than the vaporization pressure $P_{v a p}$ for the four stations near the throat.

The RMS wall pressure fluctuations are plotted in Figure 21 versus the distance $x-x_{\text {inlet }}$ for all computations. Results present large discrepancies. For small values of $M_{t_{0}}$, the peak of fluctuations is reached between station 1 and 2, and presents a plateau with a high value around 0.6-0.7. Larger is the value of $M_{t_{0}}$, larger is the abscissa of the peak location, due to the fact that the attached cavity grows. After the fifth station, RMS values decrease. For the highest value $M_{t_{0}}=2.5$, the peak of fluctuations is located between station 3 and 4, and the decrease of RMS values is close to the experimental data.

\subsubsection{Case 2}

We tested different values of $M_{t_{0}}$ from 0.1 to 0.8 . With the largest value, a very large shedding of mixture clouds appeared, which are convected very far downstream in the divergent. Figure 22 plots the contours of the density gradient modulus obtained with the original value $M_{t_{0}}=0.25$ (on the left) and a larger value $M_{t_{0}}=0.8$ (on the right). We can observe a very small at- 
tached cavity to the throat with clouds cavitation. The time-averaged cavity length is very extended with $M_{t_{0}}=0.8$ and can reach the abscissa $x=0.5 \mathrm{~m}$, whereas the length is around $0.1 \mathrm{~m}$ with $M_{t_{0}}=0.25$. With the other tested values $M_{t_{0}}=0.1$ and $M_{t_{0}}=0.5$, the time-averaged length is also around $0.1 \mathrm{~m}$ and the density gradients contours (not presented) are close to those simulated with $M_{t_{0}}=0.25$.

In the following, only two computations are presented, obtained with $M_{t_{0}}=0.25$ and $M_{t_{0}}=0.5$. Both computations predicted a periodic selfoscillating cavity but the frequency is around $20 \mathrm{~Hz}$ instead of $45 \mathrm{~Hz}$ for the experimental value (see Table 5). This frequency is similar to those obtained with the KE compressible models.

Figure 23 shows the evolution of the time-averaged longitudinal velocity (left) and the void ratio (right) for the experiments and the computations. Both models provided similar results. Velocity profiles are in close agreement with the experimental data. For the void ratio, except at station 1 where results are in good agreement, both computations overestimated the values, especially at station 3 . The clouds cavitation shedding is too extended.

The dimensionless time-averaged wall pressure distribution $\frac{P-P_{v a p}}{P_{v a p}}$ is plotted in Figure 24 versus the distance $x-x_{\text {inlet }}$. As observed with velocity and void ratio profiles, the wall pressure evolution provided by both models are quite similar and in good agreement with the experimental data. 


\subsection{Influence of the parameter of the Reboud limiter}

The limiter proposed by Reboud involves a parameter $n$, calibrated for the $k-\varepsilon$ model, and usually set to 10 for such unsteady cavitating flows. We performed different simulations with $n=10$ and a lower value $n=4$ on both geometries. Higher is the value, stronger is the reduction of the turbulent viscosity $\mu_{t}$.

\subsubsection{Case 1}

With both models, the cavitation parameter in the inlet section $\sigma_{\text {inlet }}$ is overestimated in comparison with the experimental value (see Table (5)).

The contours of the density gradient modulus are plotted in Figure 25, for the KO Reboud model with $n=4$ (left) and with $n=10$ (right). With the smallest value, we clearly observe the attached cavity up to the abscissa $x=0.025 \mathrm{~m}$, and downstream some small cavitation clouds. This situation is in correct agreement with the experimental visualizations. When the parameter $n$ is increased, the attached cavity becomes less extended (up to $x=0.015 \mathrm{~m})$, whereas the two-phase structures convected in the divergent are more developped.

Figure 26 shows the time-averaged velocity profiles (on the left) and void ratio profiles (on the right) for the experiments and both computations. We remark on the velocity profiles at station 2 that the re-entrant jet predicted with the value $n=10$ is too extended and the void ratio is largely underestimated. At stations 3, 4 and 5, results are quite similar. At the end of the cavity, the sheet thickness is too important. 
The dimensionless time-averaged wall pressure distribution $\frac{P-P_{v a p}}{P_{v a p}}$ is plotted in Figure 27 versus the distance $x-x_{\text {inlet }}$ for the experiments and both computations. With both models, the re-compression area downstream the cavity is largely under-estimated. The RMS wall pressure fluctuations are illustrated in Figure 28. With the value $n=10$, as the re-entrant jet is too extended, the peak of fluctuations is located at station 3 instead of station 5. With $n=4$ the result is improved. Downstream, both models provided a very high level of fluctuations in poor agreement with the experiment data.

\subsubsection{Case 2}

First, the frequency obtained from a DFT of the vapour volume is different between simulations (see Table (5). With $n=4$, the frequency is around $21 \mathrm{~Hz}$, whereas with $n=10$ the frequency is close to the experimental value.

The contours of the density gradient modulus are plotted in Figure 29, for the model with $n=4$ (left) and with $n=10$ (right). The sheet cavity simulated mark some difference: the attached cavity to the throat is more developped with $n=4$, whereas the two-phase structures shedding is more extended with $n=10$.

Figure 30 shows the time-averaged velocity profiles (on the left) and void ratio profiles (on the right) for the experiments and both computations. Velocity profiles are similar with both values of $n$. For the void ratio profiles, at station 1, a good agreement with the experimental data is obtained with the value $n=10$, whereas the void ratio is over-estimated (factor 3 ) with $n=4$. 
At station 2, results are quite similar. At station 3, the cavity predicted by the model with $n=4$ is too short. On the contrary, the void ratio profiles provided by the model with $n=10$ is a little over-estimated.

The dimensionless time-averaged wall pressure distribution $\frac{P-P_{\text {vap }}}{P_{\text {vap }}}$ is plotted in Figure 31 for the experiments and both computations. Both computations provided similar results, in poor agreement with the experimental data in the re-compression area.

\subsection{Partial conclusions}

A summary of results is given in Table (6). Both corrections are non universal and depends on the considered case. For the compressible model, the cut-off turbulent Mach number has to be increased to correctly compute the quasi stable cavity. On the contrary, for the cyclic cavity, high values are not adapted. As observed for the compressible $k-\varepsilon$ model, the compressible $k-\omega$ model is unable to provide the good frequency.

For the Reboud model, a smaller reduction of $\mu_{t}(n=4)$ allowed to obtain correct results for the quasi stable cavity. On the contrary, with the periodic self-oscillating case, a higher value $n=10$ allowed to predict the frequency of the phenomenon.

The level of reduction of the turbulent viscosity in the boundary layer is determinant.

\section{Conclusion}

Effects of compressibility on turbulence modelling were underlined in the mixture transport equations. Additional terms appear even if pure phases 
are assumed to be incompressible. For instance, as the divergence of the fluctuating velocity is not zero, the dilatational dissipation has to be taken into account.

From researches performed on compressible turbulence for high speed aerodynamic flows, several closure relations for these additional terms are available. In this paper, we decided to adopt ones proposed by Sarkar and Wilcox and we tested them for two different partial cavities developing on 2D Venturi configurations: an aperiodic quasi stable cavity and a self-oscillating cavity. The Reboud limiter, developped for such unsteady cases, is also tested. An impact on the value of the exponent $n$ used in this correction is performed with the $k-\omega$ Wilcox turbulence model.

Numerical results have been compared with experimental data concerning the void ratio, streamwise velocity and wall pressure.

Results showed that the compressible corrections were not adapted to simulate such cavitating flows and a recalibration is therefore necessary. For instance, both $k-\varepsilon$ and $k-\omega$ compressible models were unable to correctly predict the cyclic cavitating phenomenon. Nevertheless, the addition of dissipation terms as the dilatational dissipation allowed the development of a re-entrant jet along the wall whereas standard turbulence models always computed a stable cavity without recirculating area. Therefore, these results showed that in the mixture area, compressible effects on turbulence or at least additional dissipation effects occurred and must be included in the turbulence models. Unfortunately, due to the lack of experimental data concerning turbulent quantities in cavitating flows at high Reynolds number, it seems very difficult to calibrate and fine-tune the turbulence models. 
On another hand, the Reboud limiter, which decreases drastically the eddyviscosity in the mixture showed good agreement with the experimental data. However, tests performed with the $k-\omega$ Wilcox model exhibited an influence of the value of the parameter $n$. Indeed, to get results in agreement with the experimental measures, the value of $n$ had to be changed according to the flow configurations. Thus, the Reboud limiter can not be used to predict cavitating flows a priori, but its behaviour confirms the observations done with the compressible turbulent models and the need to include additional dissipation.

Finally, from this work, we can say that whatever the way how an additional dissipation is added in the turbulence modelling, results are improved compared with those obtained with the standard turbulence models. However, as none of the models are able to correctly computed the two cavitating flows without adjustment, additional works have to be performed to better understand the coupling between the cavitation and the turbulence and to improve the models. 
[1] J.-L. Reboud, B. Stutz, O. Coutier, Two-phase flow structure of cavitation: experiment and modelling of unsteady effects, in: $3^{\text {rd }}$ International Symposium on Cavitation CAV1998, Grenoble, France, 1998.

[2] P. Huang, G. Coleman, P. Bradshaw, Compressible turbulent channel flows: DNS results and modelling, Journal of Fluid Mechanics 305 (1995) $185-218$.

[3] R. Lechner, J. Sesterhenn, R. Friedrich, Turbulent supersonic channel flow, Journal of Turbulence 2 (1).

[4] S. Sarkar, The stabilizing effect of compressibility in turbulent shear flow, Journal of Fluid Mechanics 282 (1995) 163-186.

[5] Y. Chen, C. Lu, L. Wu, Modelling and computation of unsteady turbulent cavitation flows, Journal of Hydrodynamics 18 (5) (2006) 559-566.

[6] O. Coutier-Delgosha, R. Fortes-Patella, J. Reboud, Simulation of unsteady cavitation with a two-equation turbulence model including compressibility effects, Journal of Turbulence 3 (58).

[7] R. F. Patella, S. Barre, J. Reboud, Experiments and modelling of cavitating flows in Venturi : part II, unsteady cavitation, in: 6th International Symposium on Cavitation CAV2006, Wageningen, The Netherlands, 2006.

[8] J. Seo, S. Lele, Numerical investigation of cloud cavitation and cavitation noise on a hydrofoil section, in: $7^{\text {th }}$ International Symposium on Cavitation CAV2009, Ann Arbor, USA, 2009. 
[9] V. Srinivasan, A. Salazar, K. Saito, numerical simulation of cavitation dynamics using a cavitation-induced-momentum-defect (cimd) correction approach, Applied Mathematical Modelling 33 (2009) 1529-1559.

[10] L. Zhou, Z. Wang, Numerical simulation of cavitation around a hydrofoil and evaluation of a rng $k-\varepsilon$ model, Journal of Fluid Engineering 130 (1) (2008) 011302.

[11] F. Menter, Zonal two equation $k-\omega$ turbulence models for aerodynamic flows, in: AIAA 93-2906, 24th Fluid Dynamics Conference - Orlando, Florida, 1993.

[12] F. Menter, Two-equation eddy-viscosity turbulence models for engineering applications, AIAA Journal 32 (8) (1994) 1598-1605.

[13] P. Durbin, Limiters and wall treatments in applied turbulence modeling, Fluid Dyn. research 41 (1) (2009) 012203.

[14] E. Goncalves, Numerical study of unsteady turbulent cavitating flows, European Journal of Mechanics B/Fluids 30 (1) (2011) 26-40.

[15] D. Wilcox, Turbulence modeling for CFD, DCW Industries Inc. (1998).

[16] R. Vaidyanathan, I. Senocak, J. Wu, W. Shyy, Sensitivity evaluation of a transport-based turbulent cavitation model, Journal of Fluids Engineering 125 (5) (2003) 447-458.

[17] F. Menter, The scale-adaptive simulation mathod for unsteady turbulent flow predictions. part 1: Theory and model description, Flow, Turbulence and Combustion 85 (1) (2010) 113-138. 
[18] J. Decaix, E. Goncalves, Time-dependent simulation of cavitating flow with $k-\ell$ turbulence models, Int. Journal for Numerical Methods in Fluids 68 (2012) 1053-1072.

[19] J. Wu, G. Wang, W. Shyy, Time-dependent turbulent cavitating flow computations with interfacial transport and filter-based models, Int. Journal for Numerical Methods in Fluids 49 (7) (2005) 739-761.

[20] O. Zeman, Dilatational dissipation: the concept and application in modeling compressible mixing layers, Physic of Fluids 2 (2) (1990) 178-189.

[21] O. Zeman, New models for super/hypersonic turbulent boundary layers, in: AIAA 93-0897, 29 ${ }^{\text {th }}$ Aerospace Sciences Meeting - Reno, Nevada, 1993.

[22] S. Sarkar, G. Erlebacher, M. Hussaini, H. Kreiss, The analysis and modelling of dilatational terms in compressible turbulence, Journal of Fluid Mechanics 227 (1991) 473-493.

[23] S. Sarkar, G. Erlebacher, M. Hussaini, Compressible homogeneous shear: simulation and modeling, Tech. rep., NASA ICASE Report No 92-6 (1992).

[24] S. Sarkar, The pressure-dilatation correlation in compressible flows, Physics of Fluids 4 (12) (1992) 2674-2682.

[25] D. Wilcox, Progress in hypersonic turbulence modelling, in: AIAA 911785, 22 $2^{\text {nd }}$ Fluid Dynamic, Plasmadynamics and Laser Conference, 1991. 
[26] P. Chassaing, The modeling of variable density flows. a review of firstorder closure schemes, Flow, Turbulence and Combustion 66 (4) (2001) 293-332.

[27] T. Gatski, J.-P. Bonnet, Compressibility, turbulence and high speed flow, Elsevier (2009).

[28] S. Lele, Compressibility effects on turbulence, Annual review of Fluid Mechanics 26 (1994) 211-254.

[29] E. Goncalves, R. F. Patella, Numerical simulation of cavitating flows with homogeneous models, Computers \& Fluids 38 (9) (2009) 16821696.

[30] E. Goncalves, J. Decaix, Wall model and mesh influence study for partial cavities, European Journal of Mechanics B/Fluids 31 (1) (2012) 12-29.

[31] C. Ishii, T. Hibiki, Thermo-fluid dynamics of two-phase flow, Springer (2006).

[32] Y. Delannoy, J. Kueny, Two phase flow approach in unsteady cavitation modelling, in: Cavitation and Multiphase Flow Forum, ASME-FED, vol. 98, pp.153-158, 1990.

[33] D. Wilcox, Reassement of the scale-determining equation for advanced turbulence models, AIAA Journal 26 (11) (1988) 1299-1310.

[34] W. Jones, B. Launder, The prediction of laminarization with a twoequation model of turbulence, Int. J. Heat Mass Transfer 15 (1972) $301-314$. 
[35] O. Zeman, On the decay of compressible isotropic turbulence, Physic of Fluids 3 (5) (1991) 951-955.

[36] M. Rubesin, Tech. rep., NASA TM X-73,128 (1990).

[37] W. Jones, Prediction methods for turbulent flows, in: Von Karman Institute, Lecture Series 1979-2, 1979.

[38] S. Sarkar, L. Balakrishnan, Application of a reynolds stress turbulence model to the compressible shear layer, Tech. rep., NASA ICASE Report No 90-18 (1990).

[39] S. Sarkar, G. E. M. Hussaini, H. Kreiss, The analysis and modeling of dilatational terms in compressible turbulence, Tech. rep., NASA ICASE Report No 89-79 (1989).

[40] D. Wilcox, Dilatation-dissipation corrections for advances models, AIAA Journal 306 (11) (1992) 2639-2646.

[41] D. Besnard, F. Harlow, Turbulence in multiphase flow, Int. Journal of Multiphase Flow 14 (6) (1988) 679-699.

[42] S. Elghobashi, T. Abou-Arab, A two-equation turbulence model for twophase flows, Physics of Fluids 26 (4) (1983) 931-938.

[43] I. Kataoka, A. Serizawa, Basic equations of turbulence in gas-liquid two-phase flow, International Journal of Multiphase Flow 15 (5) (1989) 843-855.

[44] M. Lance, J. MArie, J. Bataille, Construction of a model for liquid phase turbulence in bubble flow, La Houille Blanche 3-4 (1984) 255-260. 
[45] E. Turkel, Preconditioned methods for solving the incompressible and low speed compressible equations, Journal of Computational Physics 172 (2) (1987) 277-298.

[46] A. Jameson, W. Schmidt, E. Turkel, Numerical solution of the Euler equations by finite volume methods using Runge-Kutta time stepping schemes, in: AIAA Paper 81-1259, 1981.

[47] P. Roe, Approximate Riemann solvers, parameters vectors, and difference schemes, Journal of Computational Physics 43 (1981) 357-372.

[48] S. Tatsumi, L. Martinelli, A. Jameson, Flux-limited schemes for the compressible Navier-Stokes equations, AIAA Journal 33 (2) (1995) 252261.

[49] H. Luo, J. Baum, R. Lohner, A fast, matrix-free implicit method for compressible flows on unstructured grids, Journal of Computational Physics 146 (2) (1998) 664-690.

[50] B. Merci, J. Steelant, J. Vierendeels, K. Riemslagh, E. Dick, Computational treatment of source terms in two-equation turbulence models, AIAA Journal 38 (11) (2000) 2085-2093.

[51] A. Jameson, Time dependent calculations using multigrid with applications to unsteady flows past airfoils and wings, in: AIAA paper 91-1259, $10^{\text {th }}$ Computational Fluid Dynamics Conference, Honolulu (HI), 1991.

[52] S. Barre, J. Rolland, G. Boitel, E. Goncalves, R. F. Patella, Experiments and modelling of cavitating flows in Venturi: attached sheet cavitation, European Journal of Mechanics B/Fluids 28 (2009) 444-464. 
Table 1: Geometrical data and flow configuration, Venturi geometries

\begin{tabular}{lcc} 
experimental parameters & case 1 & case 2 \\
\hline angle of the divergent & $4^{\circ}$ & $8^{\circ}$ \\
reference length $L_{r e f}$ & $252 \mathrm{~mm}$ & $224 \mathrm{~mm}$ \\
inlet section $S_{i}$ & $50 \times 44 \mathrm{~mm}^{2}$ & $50 \times 44 \mathrm{~mm}^{2}$ \\
\hline inlet velocity $U_{\text {inlet }}$ & $10.8 \mathrm{~m} / \mathrm{s}$ & $7.04 \mathrm{~m} / \mathrm{s}$ \\
inlet pressure $P_{\text {inlet }}$ & $0.35 \mathrm{bar}$ & $0.52 \mathrm{bar}$ \\
reference temperature & $\simeq 293 \mathrm{~K}$ & $\simeq 293 \mathrm{~K}$ \\
cavitation parameter in the inlet section $\sigma_{\text {inlet }}$ & $0.547 \pm 0.05$ & $2.15 \pm 0.06$ \\
Reynolds number $R e_{L_{r e f}}$ & $2.710^{6}$ & $1.5710^{6}$ \\
\hline sheet cavity & smoothly fluctuating & self-oscillating \\
clouds shedding frequency & none & $45 \mathrm{~Hz}$ \\
time-averaged cavity length & $80 \mathrm{~mm}$ & -
\end{tabular}


Table 2: Numerical data and meshes

\begin{tabular}{lcc} 
numerical parameters & case 1 & case 2 \\
\hline meshes & $251 \times 62$ & $174 \times 56$ \\
\hline$y^{+}$values in first cells & 12 to 27 & 9 to 31 \\
\hline preconditioning parameter & 4 & 6 \\
CFL number & 0.5 & 0.3 \\
implicit Jacobi iterations & 15 & 15 \\
2nd and 4th order dissipation parameter & $1 ; 0.045$ & $1 ; 0.055$ \\
dual time stepping sub-iterations & 100 & 100 \\
dimensionless time step, $\Delta t^{*}=\frac{\Delta t U_{\text {inlet }}}{L_{r e f}}$ & $9.7510^{-3}$ & $1.2710^{-4}$ \\
\hline farfield value of turbulent kinetic energy & $0.0045 \mathrm{~m}^{2} / \mathrm{s}^{2}$ & $0.0045 \mathrm{~m}^{2} / \mathrm{s}^{2}$ \\
farfield value of dissipation rate & $17 \mathrm{~m}^{2} / \mathrm{s}^{3}$ & $17 \mathrm{~m}^{2} / \mathrm{s}^{3}$ \\
farfield value of specific dissipation & $45000 \mathrm{~s}^{-1}$ & $45000 \mathrm{~s}^{-1}$ \\
\hline
\end{tabular}


Table 3: Unsteady computations performed with the Jones-Launder $k-\varepsilon$ model.

\begin{tabular}{|c|c|c|c|}
\hline case & turb. model & $\sigma_{\text {inlet }}$ & sheet length $(\mathrm{m})$ or frequency $(\mathrm{Hz})$ \\
\hline expe & - & 0.55 & $0.08 \mathrm{~m}$ \\
\hline 1 & KE compressible & 0.64 & $0.084 \mathrm{~m}$ \\
\hline 1 & $\mathrm{KE} \operatorname{mass} \sigma_{p}=1$ & 0.63 & $0.080 \mathrm{~m}$ \\
\hline 1 & $\mathrm{KE}$ mass $\sigma_{p}=0.001$ & 0.63 & $0.080 \mathrm{~m}$ \\
\hline 1 & $\mathrm{KE} \mathrm{pd} \alpha_{3}=0.025$ & 0.635 & $0.085 \mathrm{~m}$ \\
\hline 1 & $\mathrm{KE} \mathrm{pd} \alpha_{3}=0.001$ & 0.64 & $0.18 \mathrm{~m}$ \\
\hline 1 & $\mathrm{KE}$ epsd $\alpha_{1}=0.5$ & 0.63 & $0.22 \mathrm{~m}$ \\
\hline 1 & $\mathrm{KE}$ epsd $\alpha_{1}=0.05$ & 0.635 & $0.09 \mathrm{~m}$ \\
\hline 1 & $\mathrm{KE}$ epsd $\alpha_{1}=0.01$ & 0.635 & $0.087 \mathrm{~m}$ \\
\hline 1 & KE Reboud & 0.56 & $0.077 \mathrm{~m}$ \\
\hline expe & - & $2.15 \pm 0.06$ & $45 \mathrm{~Hz}$ \\
\hline 2 & KE compressible & 2.09 & $19 \mathrm{~Hz}$ \\
\hline 2 & $\mathrm{KE} \mathrm{pd} \alpha_{3}=0.025$ & 2.10 & $21 \mathrm{~Hz}$ \\
\hline 2 & $\mathrm{KE}$ epsd $\alpha_{1}=0.05$ & 2.09 & $20 \mathrm{~Hz}$ \\
\hline 2 & KE Reboud & 2.09 & $43 \mathrm{~Hz}$ \\
\hline
\end{tabular}


Table 4: Constants influence on the formulation of the compressible Jones-Launder $k-\varepsilon$ model, $4^{\circ}$ Venturi.

\begin{tabular}{llll}
\hline additional term & constant & attached sheet length & re-entrant jet \\
\hline mass flux & $\sigma_{p}=1$ & $0.060 \mathrm{~m}$ & not enough extended \\
& $\sigma_{p}=0.001$ & $0.060 \mathrm{~m}$ & not enough extended \\
\hline pressure-dilatation & $\alpha_{3}=0.2$ & diverge & diverge \\
& $\alpha_{3}=0.05$ & $0.06 \mathrm{~m}$ & not enough extended \\
& $\alpha_{3}=0.025$ & $0.025 \mathrm{~m}$ & extended \\
& $\alpha_{3}=0.001$ & $0.02 \mathrm{~m}$ & very extended \\
\hline extra-dissipation & $\alpha_{1}=0.5$ & $0.02 \mathrm{~m}$ & very extended \\
& $\alpha_{1}=0.05$ & $0.04 \mathrm{~m}$ & extended \\
& $\alpha_{1}=0.01$ & $0.065 \mathrm{~m}$ & not enough extended \\
\hline
\end{tabular}


Table 5: Unsteady computations performed with the Wilcox $k-\omega$ model.

\begin{tabular}{lllc}
\hline case & turb. model & $\sigma_{\text {inlet }}$ & sheet length $(\mathrm{m})$ or frequency $(\mathrm{Hz})$ \\
\hline expe & - & 0.55 & $0.08 \mathrm{~m}$ \\
1 & KW comp. $M_{t} 0=0.25$ & 0.645 & $0.077 \mathrm{~m}$ \\
1 & KW comp. $M_{t} 0=0.8$ & 0.64 & $0.078 \mathrm{~m}$ \\
1 & KW comp. $M_{t} 0=1.5$ & 0.64 & $0.076 \mathrm{~m}$ \\
1 & KW comp. $M_{t} 0=2.5$ & 0.64 & $0.073 \mathrm{~m}$ \\
1 & KW Reboud $n=10$ & 0.62 & $0.082 \mathrm{~m}$ \\
1 & KW Reboud $n=4$ & 0.625 & $0.080 \mathrm{~m}$ \\
\hline expe & - & $2.15 \pm 0.06$ & $45 \mathrm{~Hz}$ \\
2 & KW comp. $M_{t} 0=0.25$ & 2.10 & $20 \mathrm{~Hz}$ \\
2 & KW comp. $M_{t} 0=0.5$ & 2.09 & $18 \mathrm{~Hz}$ \\
2 & KW Reboud $n=10$ & 2.09 & $41 \mathrm{~Hz}$ \\
2 & KW Reboud $n=4$ & 2.08 & $21 \mathrm{~Hz}$ \\
\hline
\end{tabular}


Table 6: Summary, Wilcox model.

\begin{tabular}{lll}
\hline case & Wilcox compressible & Wilcox + Reboud \\
\hline quasi stable cavity & $M_{t_{0}}=0.25$ is not adapted, & $n=10$ is not adapted. \\
& must be increased to 2.5 & $n=4$ gives correct results. \\
\hline periodic cavity & $M_{t_{0}}=0.25$ gives correct profiles & $n=10$ gives correct profiles \\
& but frequency not predicted. & and good frequency. \\
& & $n=4$ is not adapted. \\
\hline
\end{tabular}



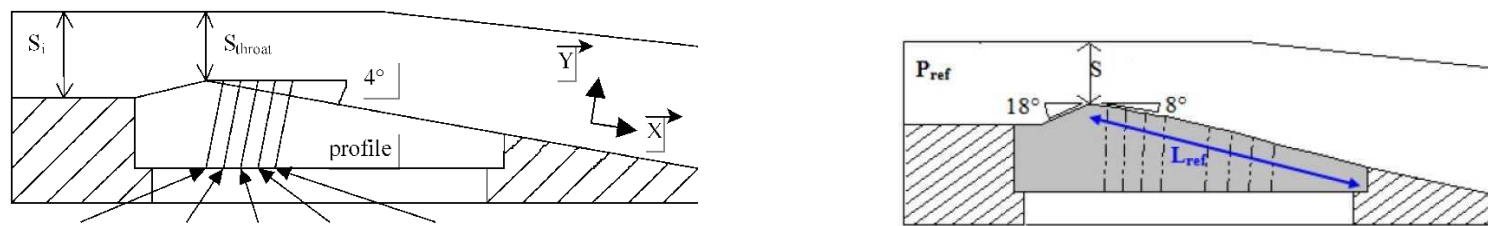

Figure 1: Venturi design and probe locations, $4^{\circ}$ divergence angle (left) and $8^{\circ}$ angle (right). 

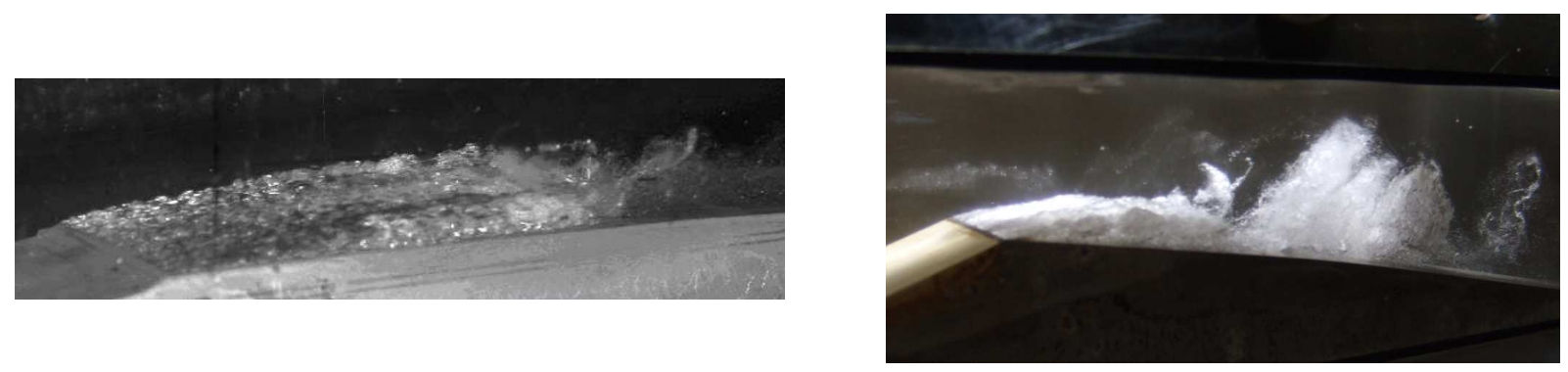

Figure 2: Photograph of cavities, $4^{\circ}$ Venturi (left) and $8^{\circ}$ Venturi (right). 


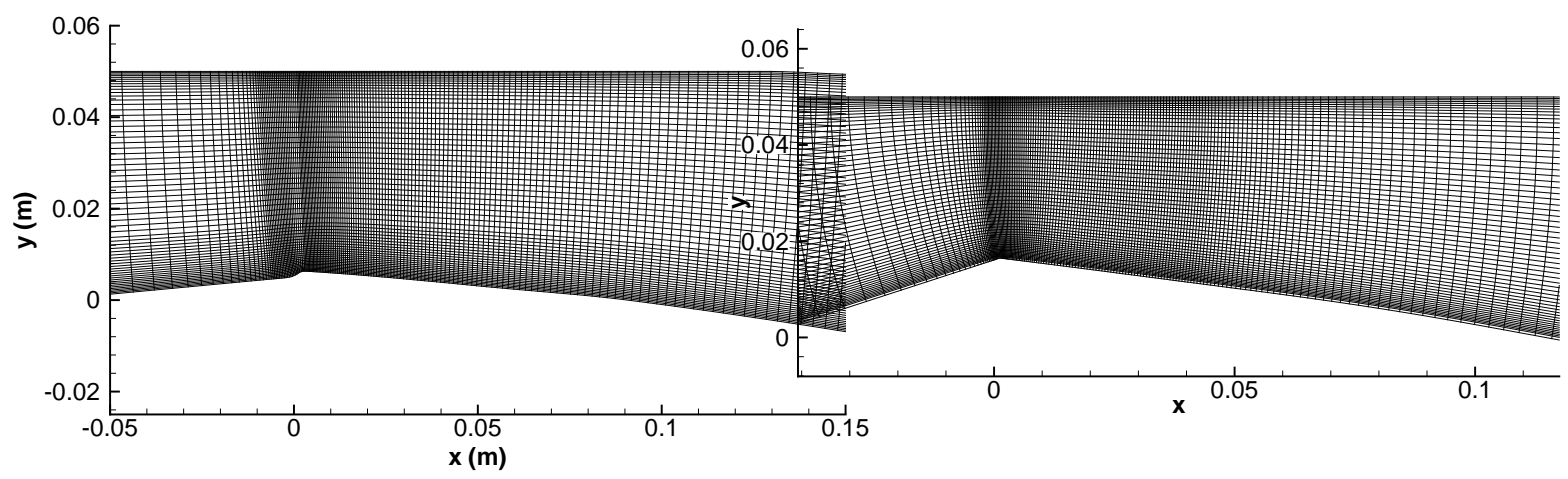

Figure 3: Enlargement of the mesh near the Venturi throat, $4^{\circ}$ Venturi (left) and $8^{\circ}$ Venturi (right). 


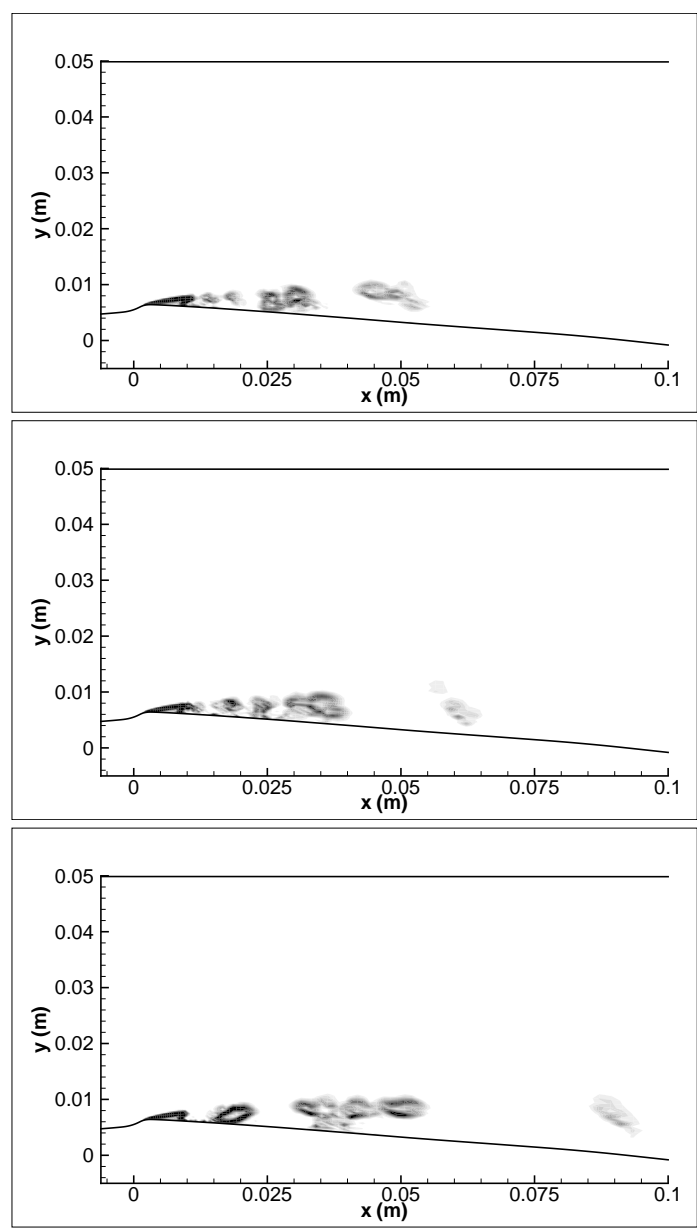

Figure 4: Contours of the density gradient modulus at three instants, KE compressible, $4^{\circ}$ Venturi. 


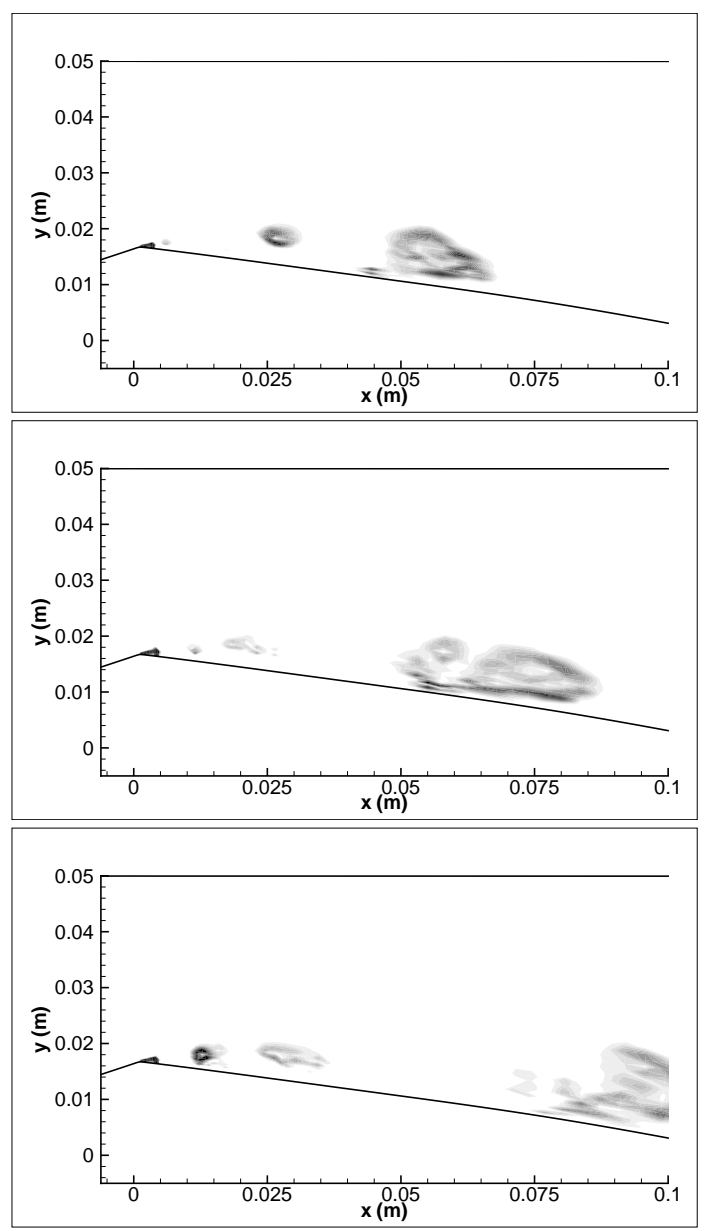

Figure 5: Contours of the density gradient modulus during one period, KE compressible, $8^{\circ}$ Venturi. 

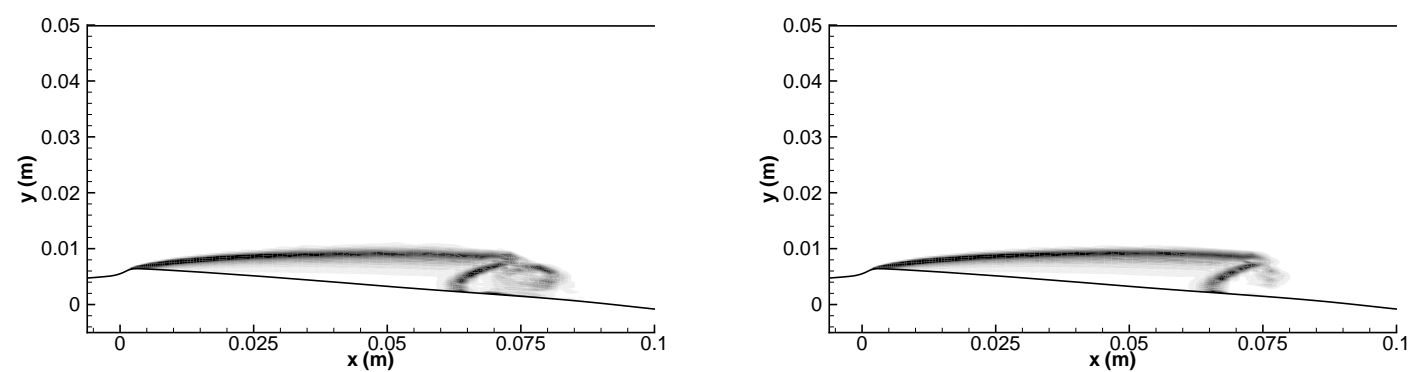

Figure 6: Contours of the instantaneous density gradient modulus, KE mass, $\sigma_{p}=1$ (left) versus $\sigma_{p}=0.001$ (right), $4^{\circ}$ Venturi. 


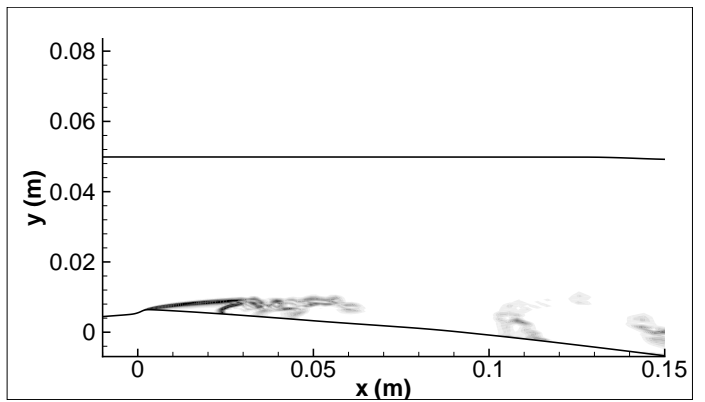

Figure 7: Contour of the instantaneous density gradient modulus, $\mathrm{KE} p^{\prime} d^{\prime}, \alpha_{3}=0.001,4^{\circ}$ Venturi. 

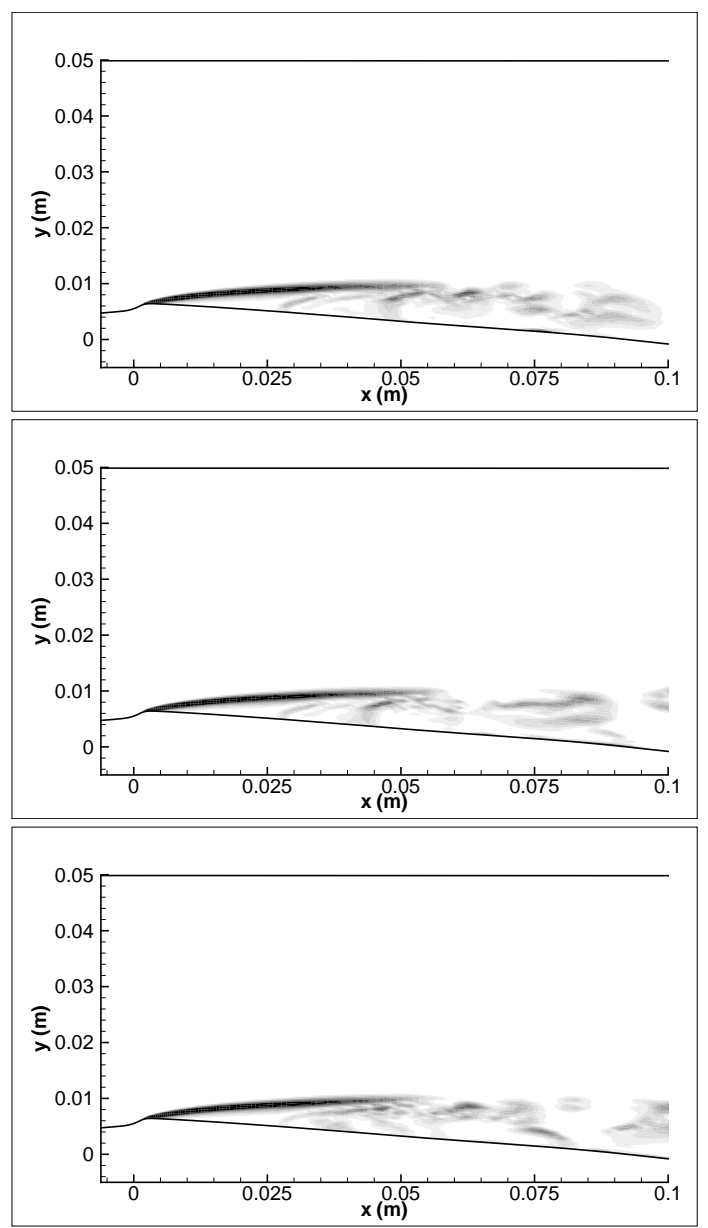

Figure 8: Contours of the density gradient modulus at three instants, $\mathrm{KE} p^{\prime} d^{\prime}, \alpha_{3}=0.025$, $4^{\circ}$ Venturi. 


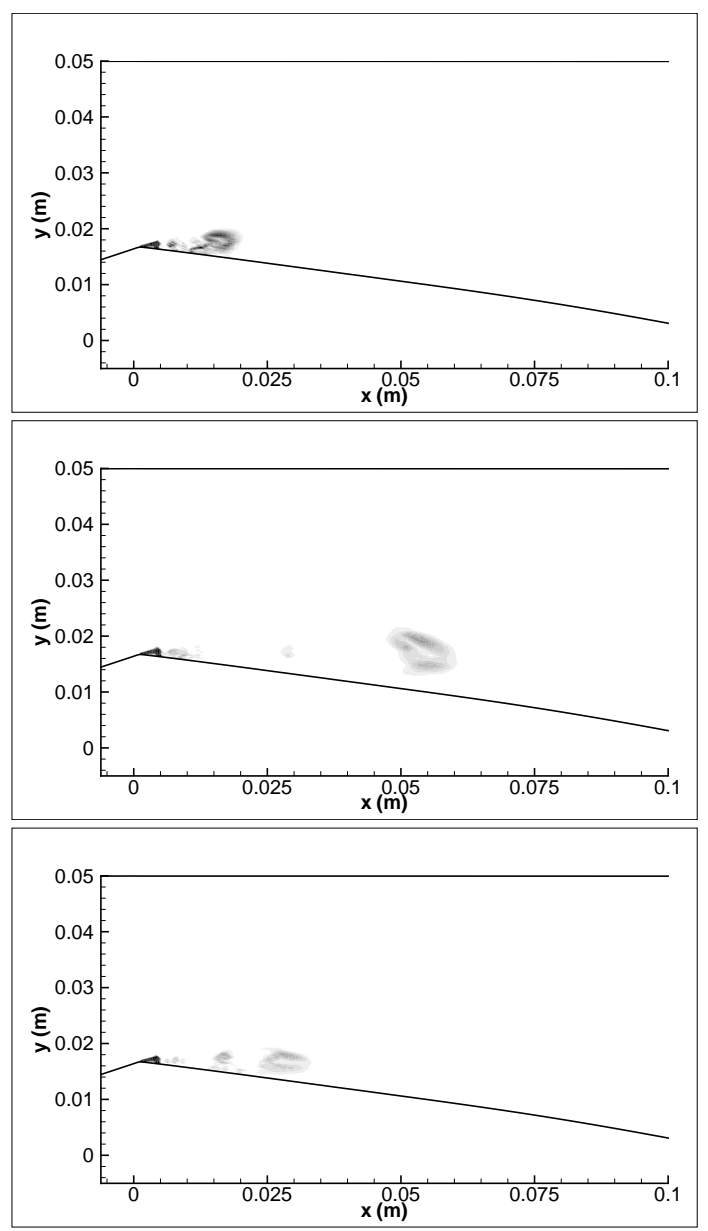

Figure 9: Contours of the density gradient modulus during one period, $\operatorname{KE} p^{\prime} d^{\prime}, \alpha_{3}=0.025$, $8^{\circ}$ Venturi. 

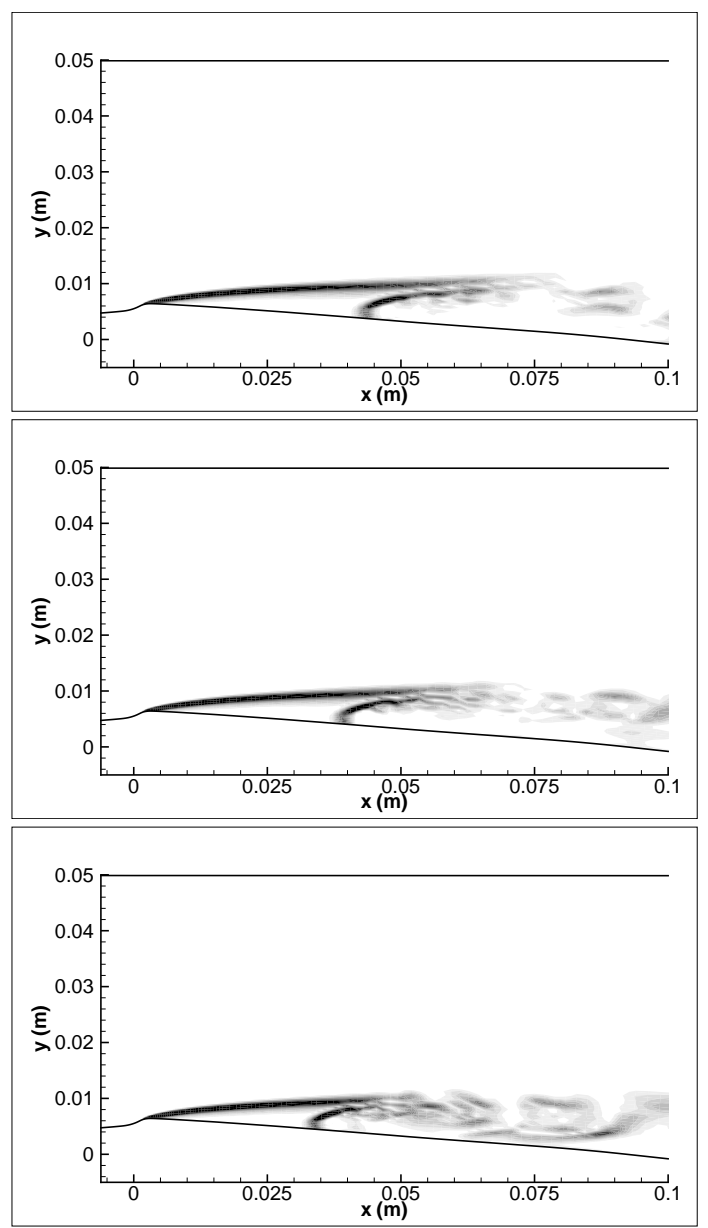

Figure 10: Contours of the density gradient modulus at three instants, $\operatorname{KE} \varepsilon_{d}, \alpha_{1}=0.05$, $4^{\circ}$ Venturi. 


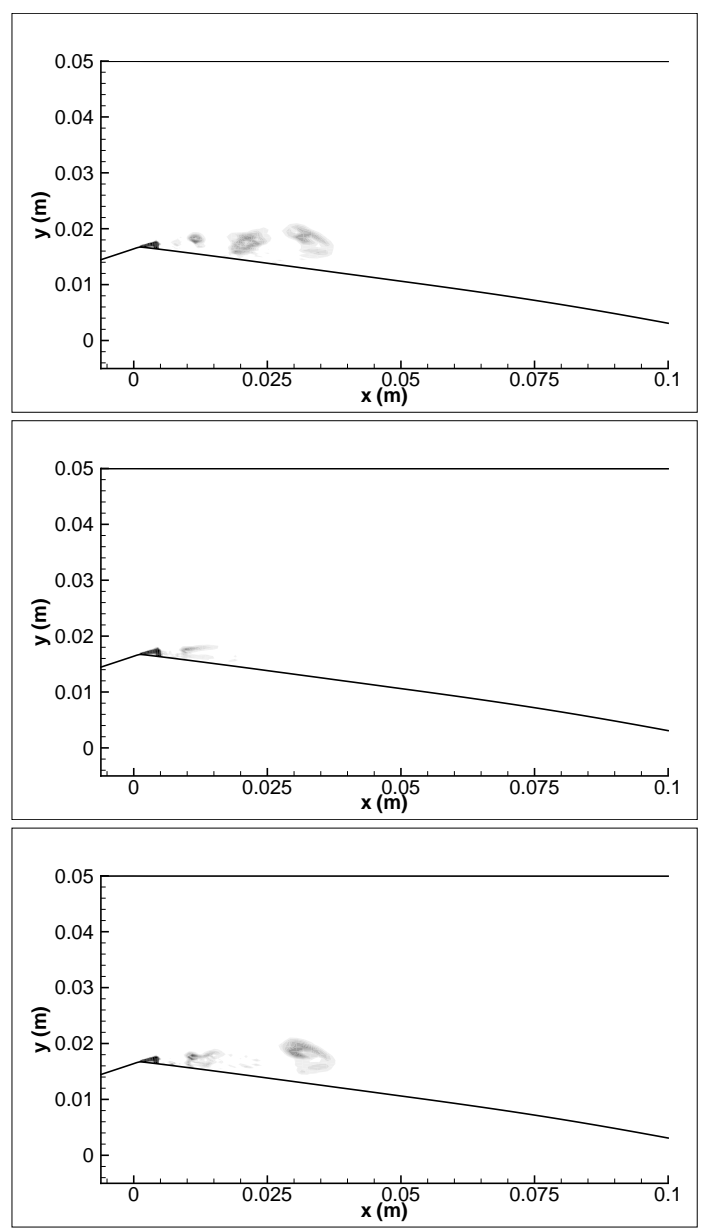

Figure 11: Contours of the density gradient modulus during one period, $\mathrm{KE} \varepsilon_{d}, \alpha_{1}=0.05$, $8^{\circ}$ Venturi. 

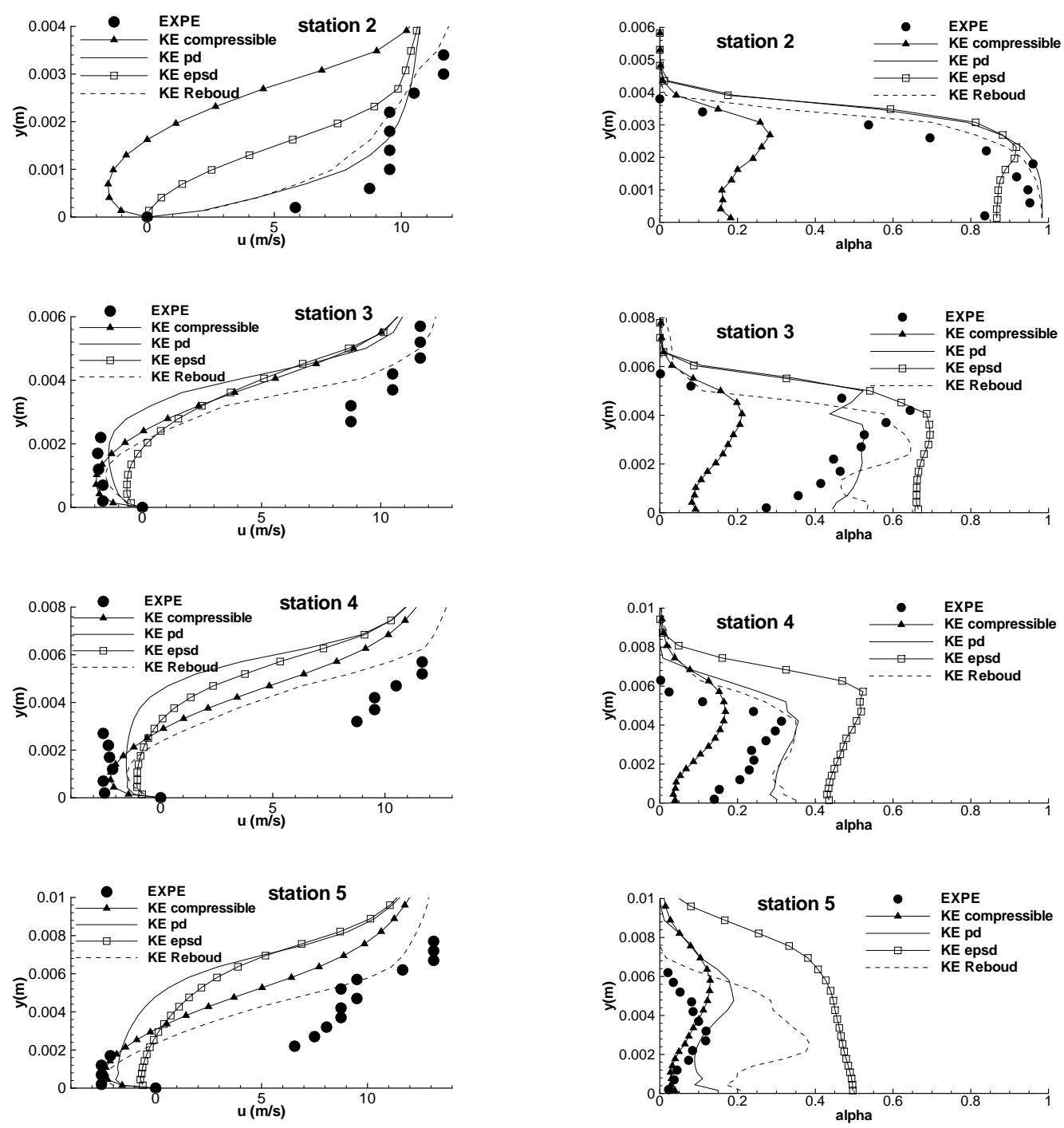

Figure 12: Time-averaged velocity (left) and void ratio (right) profiles from station 2 to 5, KE models, $4^{\circ}$ Venturi. 


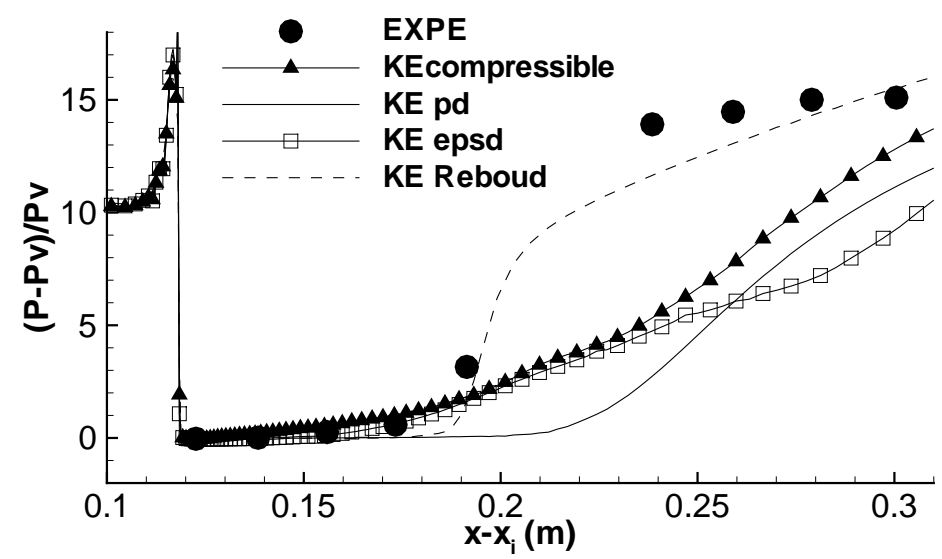

Figure 13: Dimensionless time-averaged wall pressure evolution, KE models, $4^{\circ}$ Venturi. 


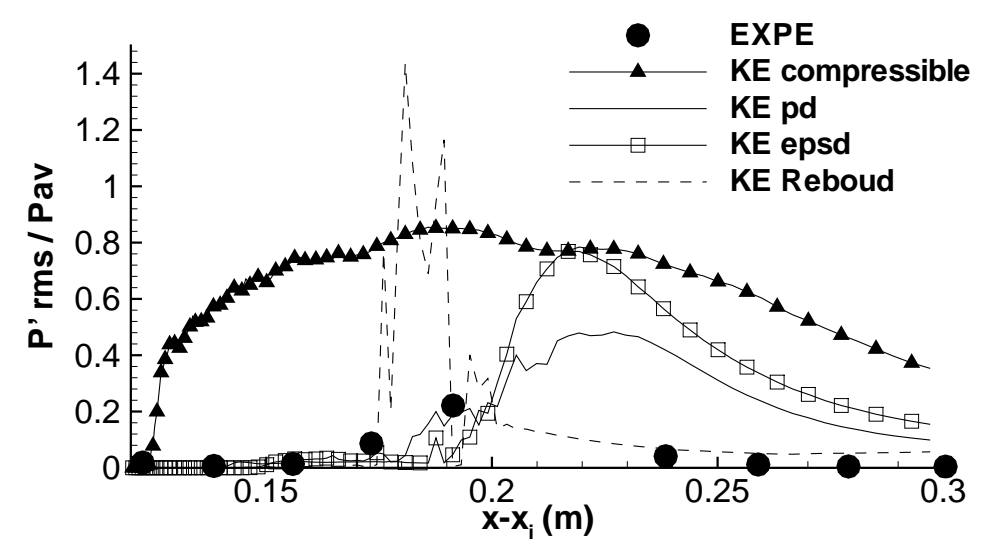

Figure 14: RMS wall pressure fluctuations, KE models, $4^{\circ}$ Venturi. 

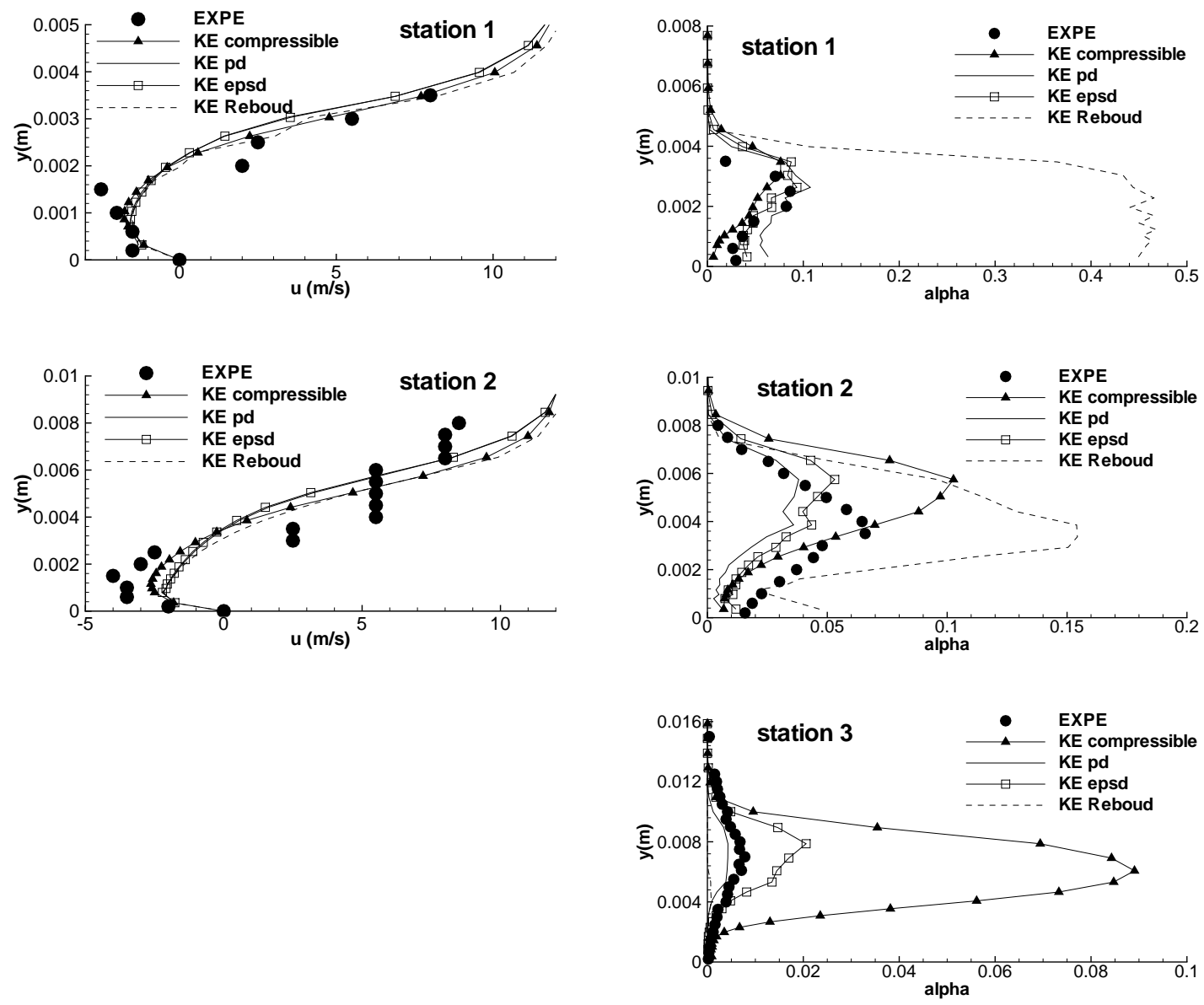

Figure 15: Time-averaged velocity (left) and void ratio (right) profiles from station 1 to

3 , KE models, $8^{\circ}$ Venturi. 


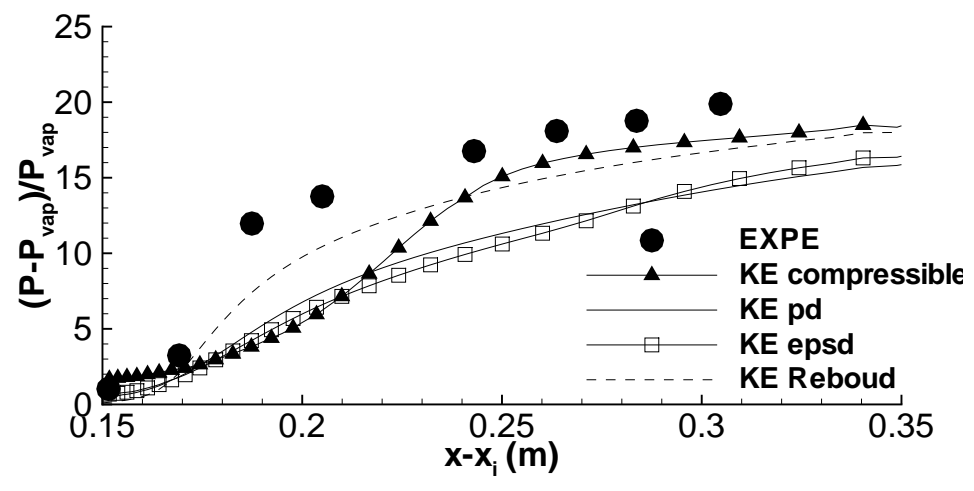

Figure 16: Dimensionless time-averaged wall pressure evolution, KE models, $8^{\circ}$ Venturi. 

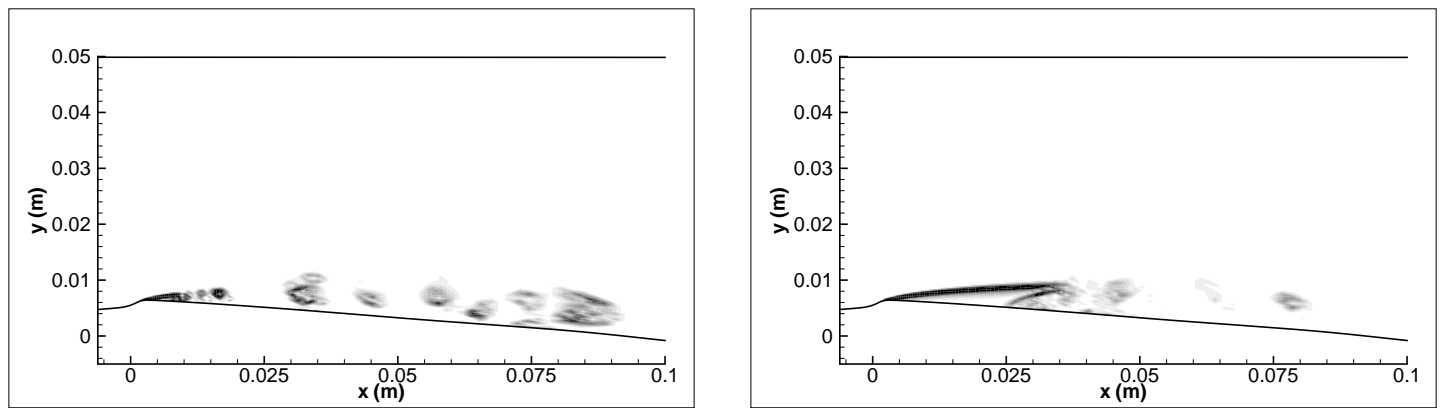

Figure 17: Contours of the instantaneous density gradient modulus, KO compressible, $M_{t_{0}}=0.25$ (left) versus $M_{t_{0}}=2.5$ (right), $4^{\circ}$ Venturi. 

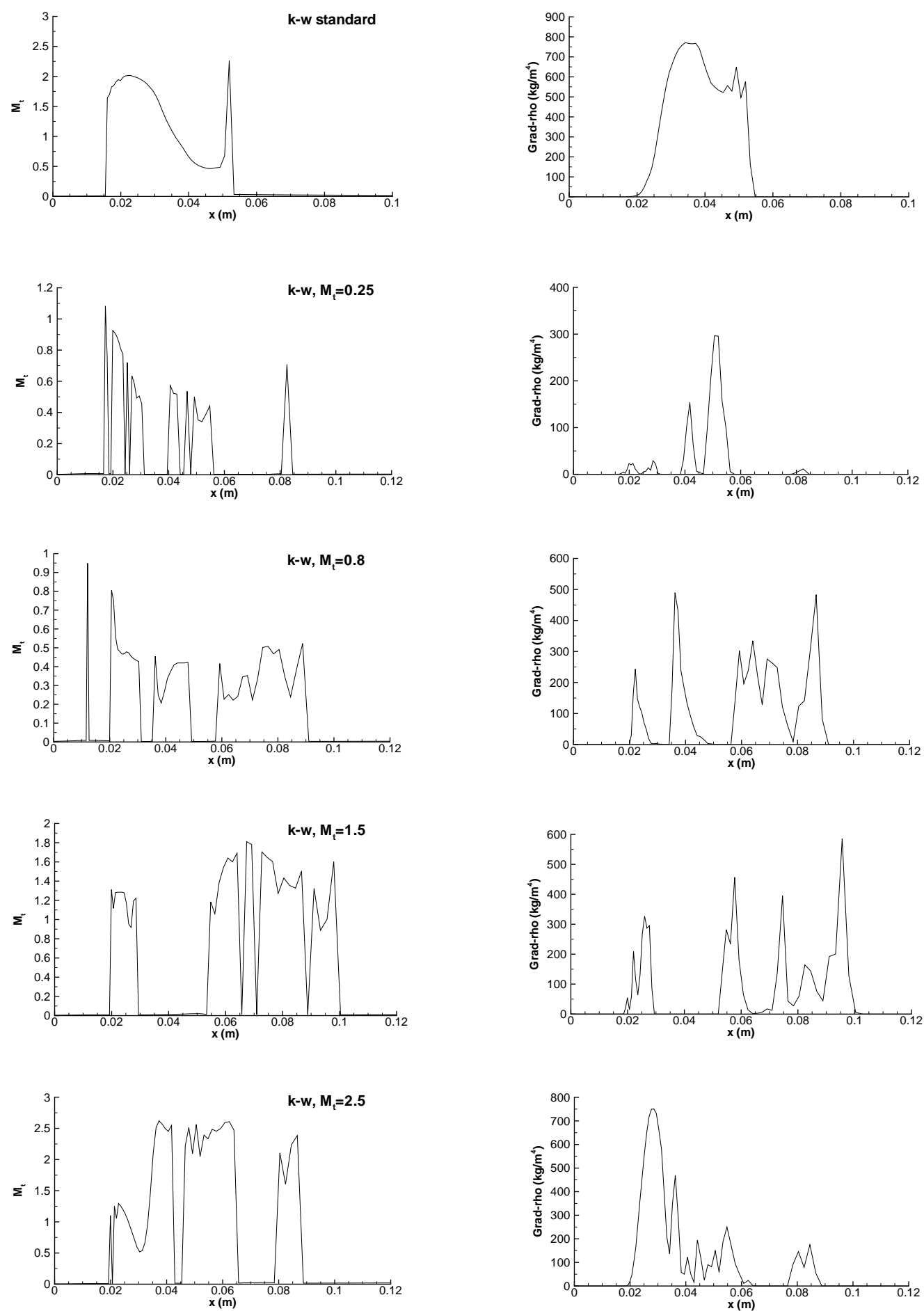

Figure 18: Instantaneous values of the turibulent Mach number (left) and the density gradient modulus (right) versus the abscissa $x$, line $j=12$, Wilcox models, $4^{\circ}$ Venturi. 

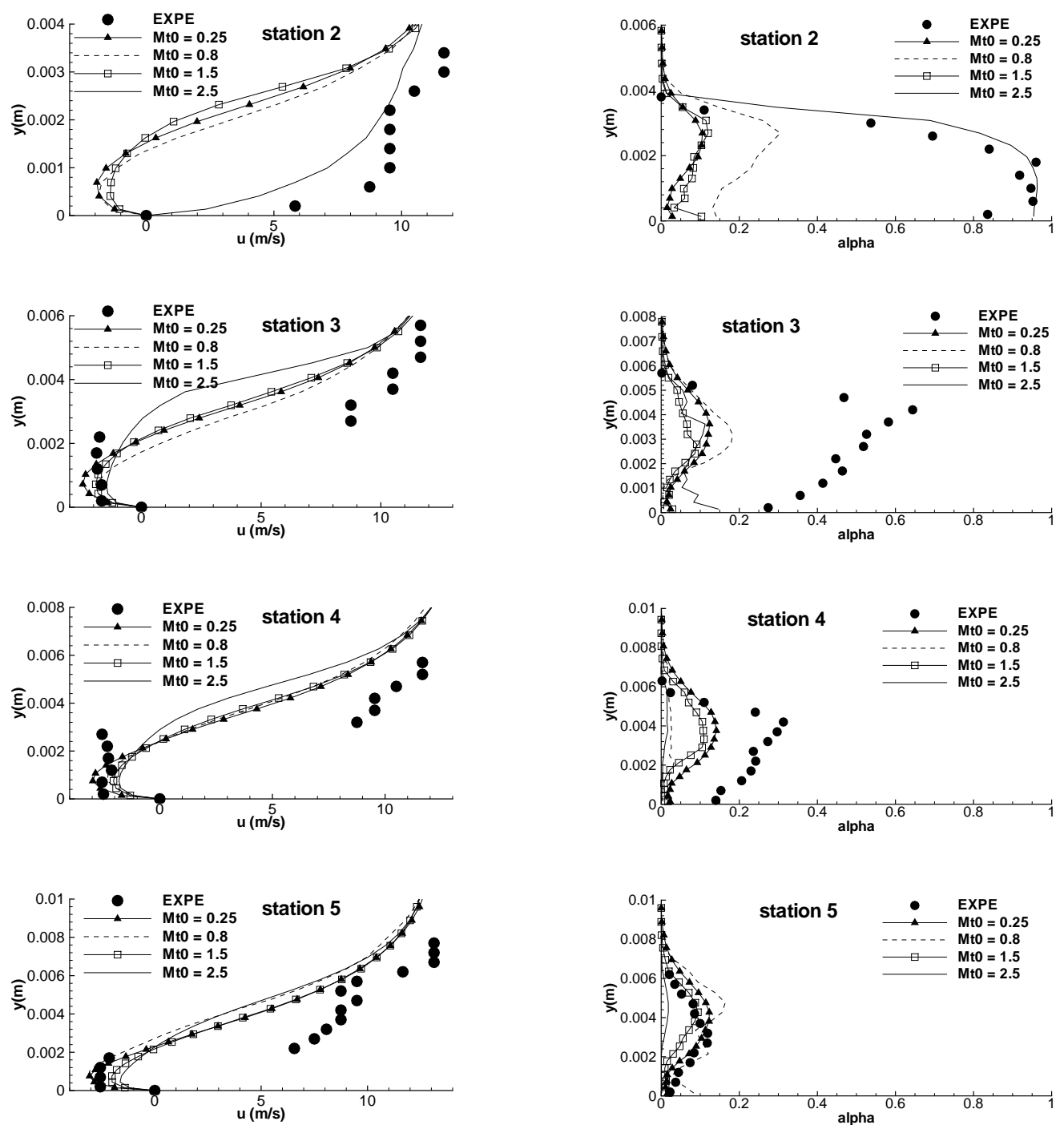

Figure 19: Time-averaged velocity (left) and void ratio (right) profiles from station 2 to 5, Wilcox compressible model, $4^{\circ}$ Venturi. 


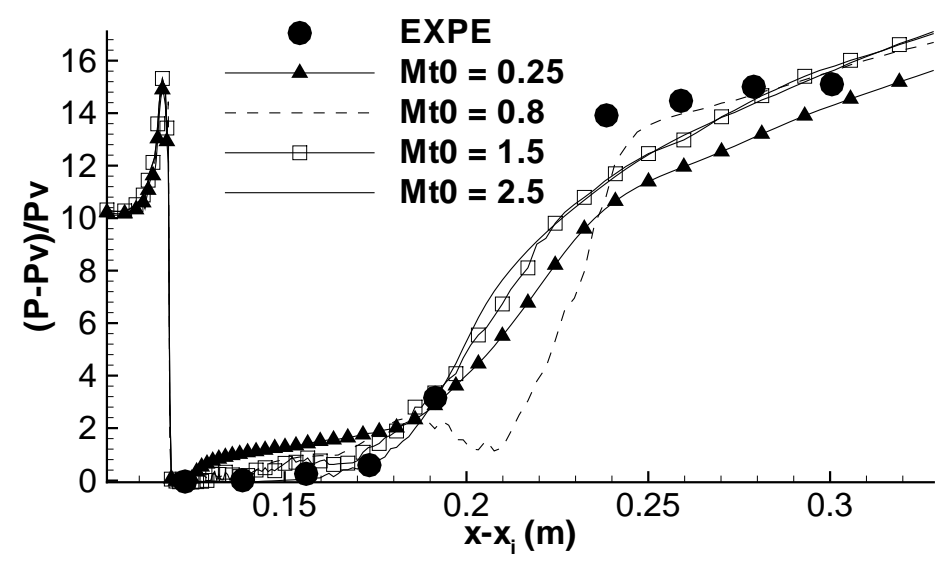

Figure 20: Dimensionless time-averaged wall pressure evolution, KO compressible models, $4^{\circ}$ Venturi. 


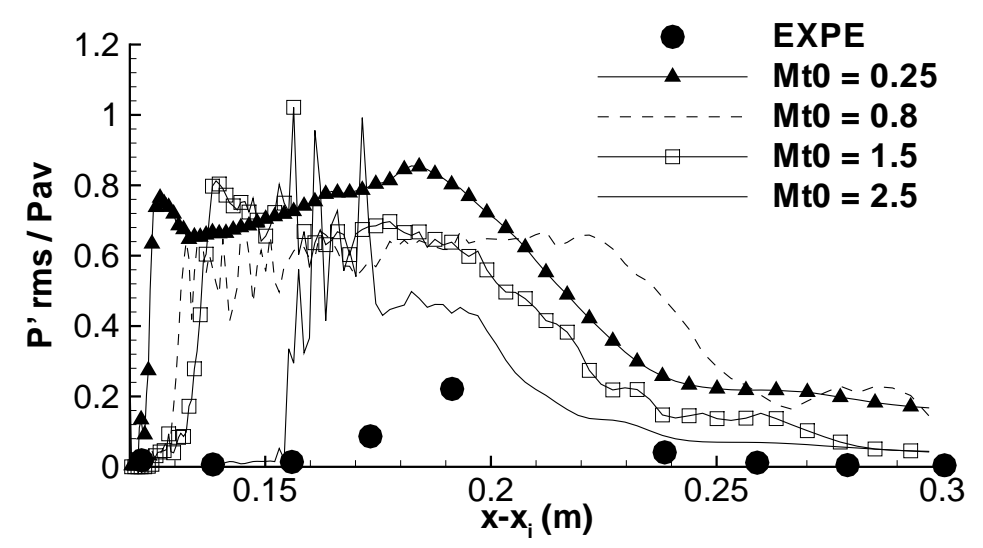

Figure 21: RMS wall pressure fluctuations, KO compressible models, $4^{\circ}$ Venturi. 

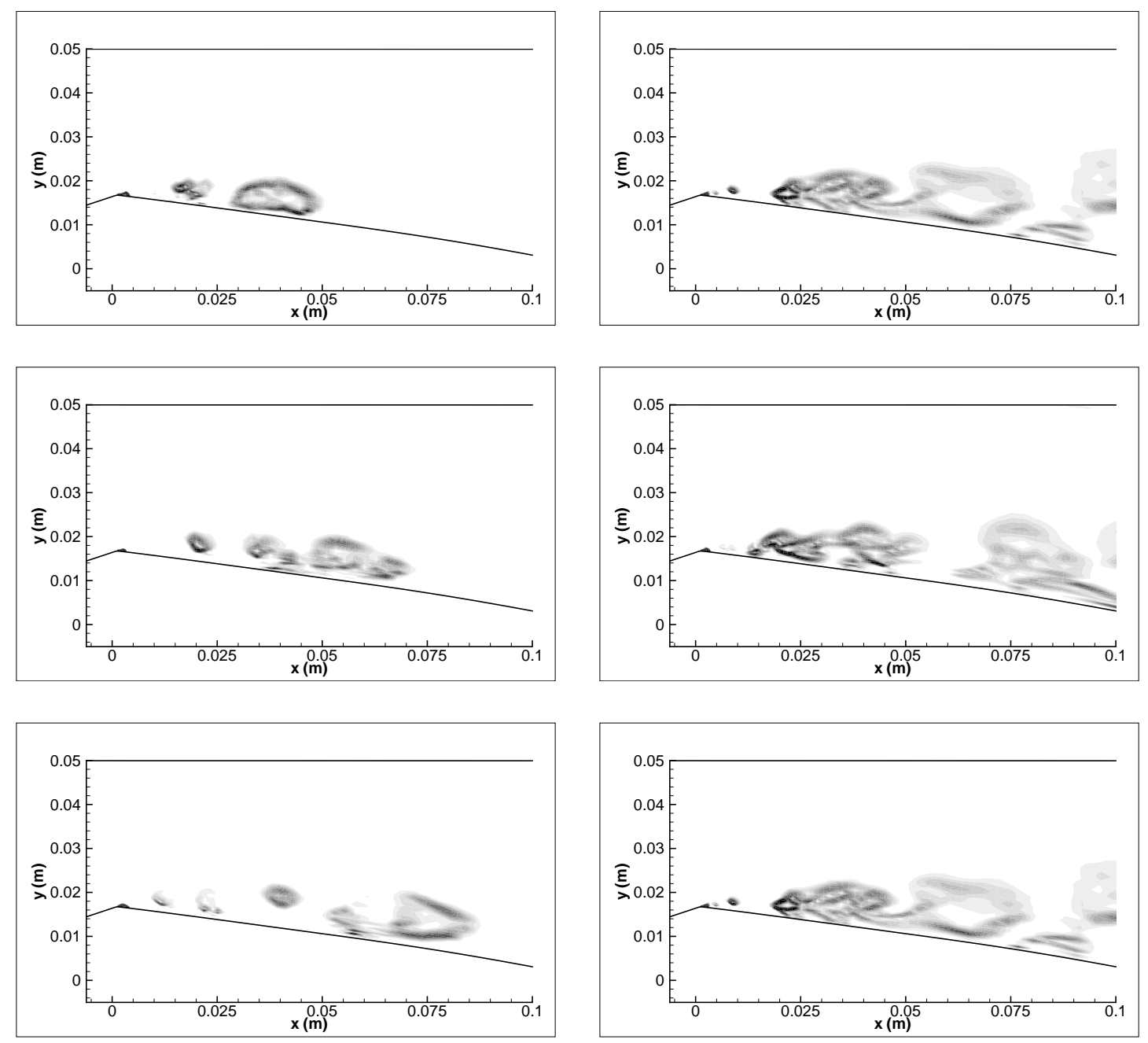

Figure 22: Contours of the density gradient modulus during one period, $\mathrm{KO}$ compressible models, $M_{t_{0}}=0.25$ (left) and $M_{t_{0}}=0.8$ (right), $8^{\circ}$ Venturi. 

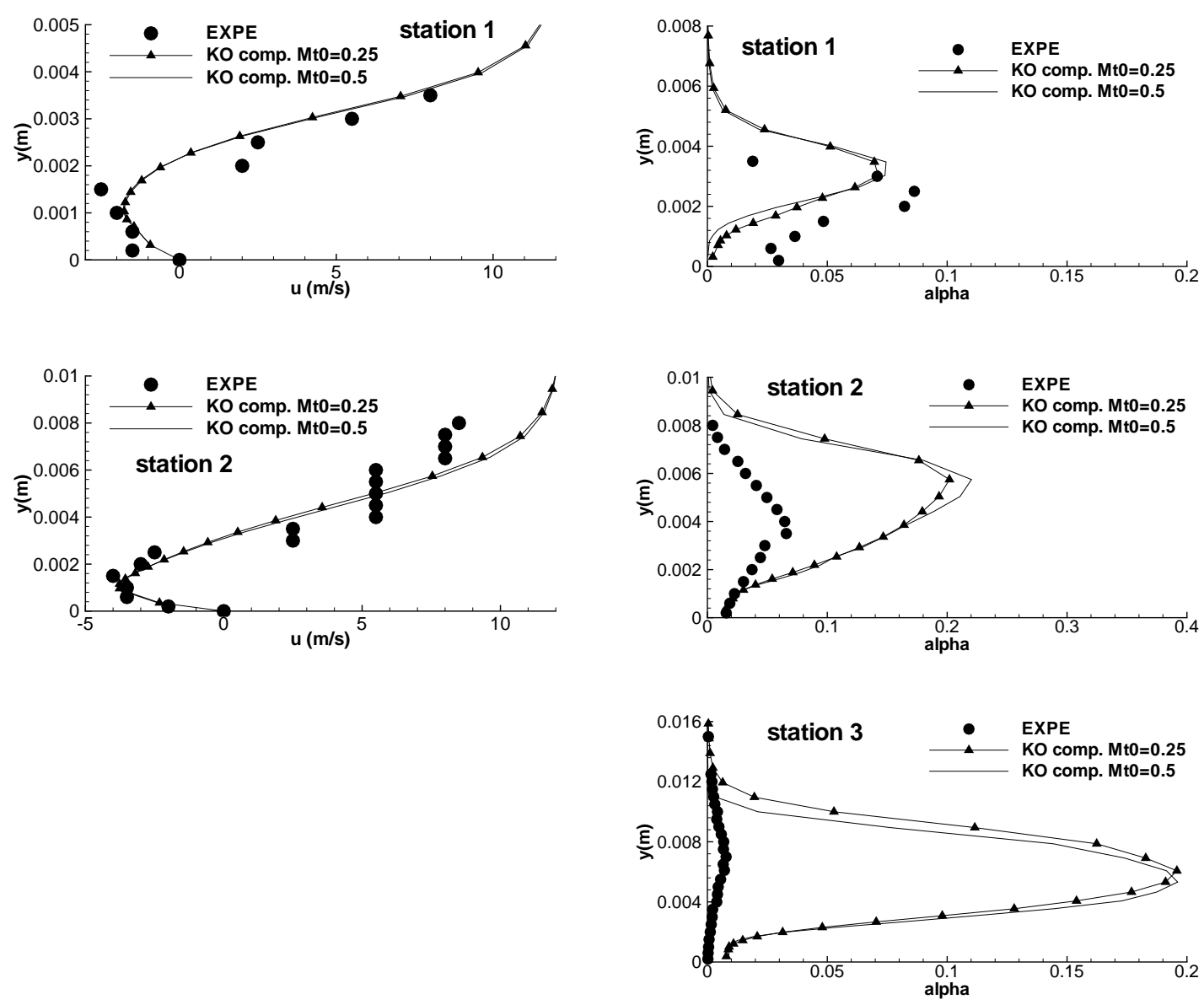

Figure 23: Velocity (left) and void ratio (right) profiles from station 1 to $3, \mathrm{KO}$ compressible model, $8^{\circ}$ Venturi. 


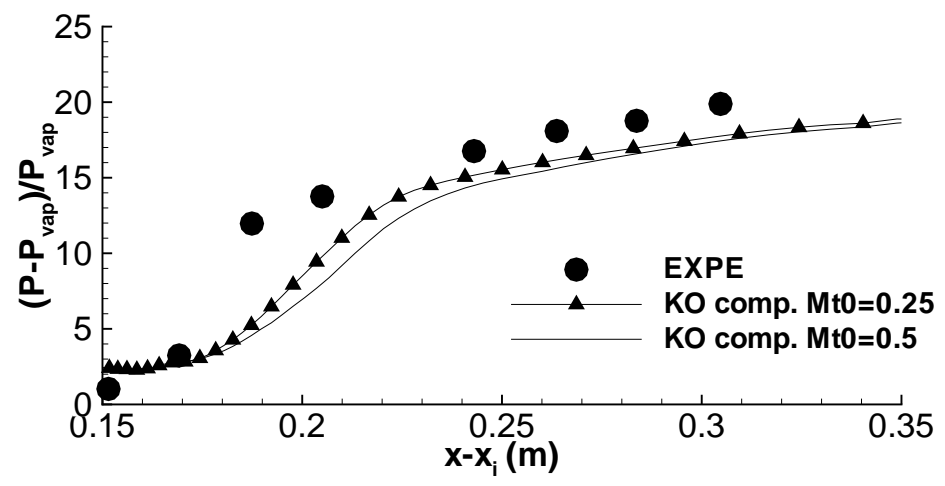

Figure 24: Dimensionless wall pressure evolution, KO compressible model, $8^{\circ}$ Venturi. 

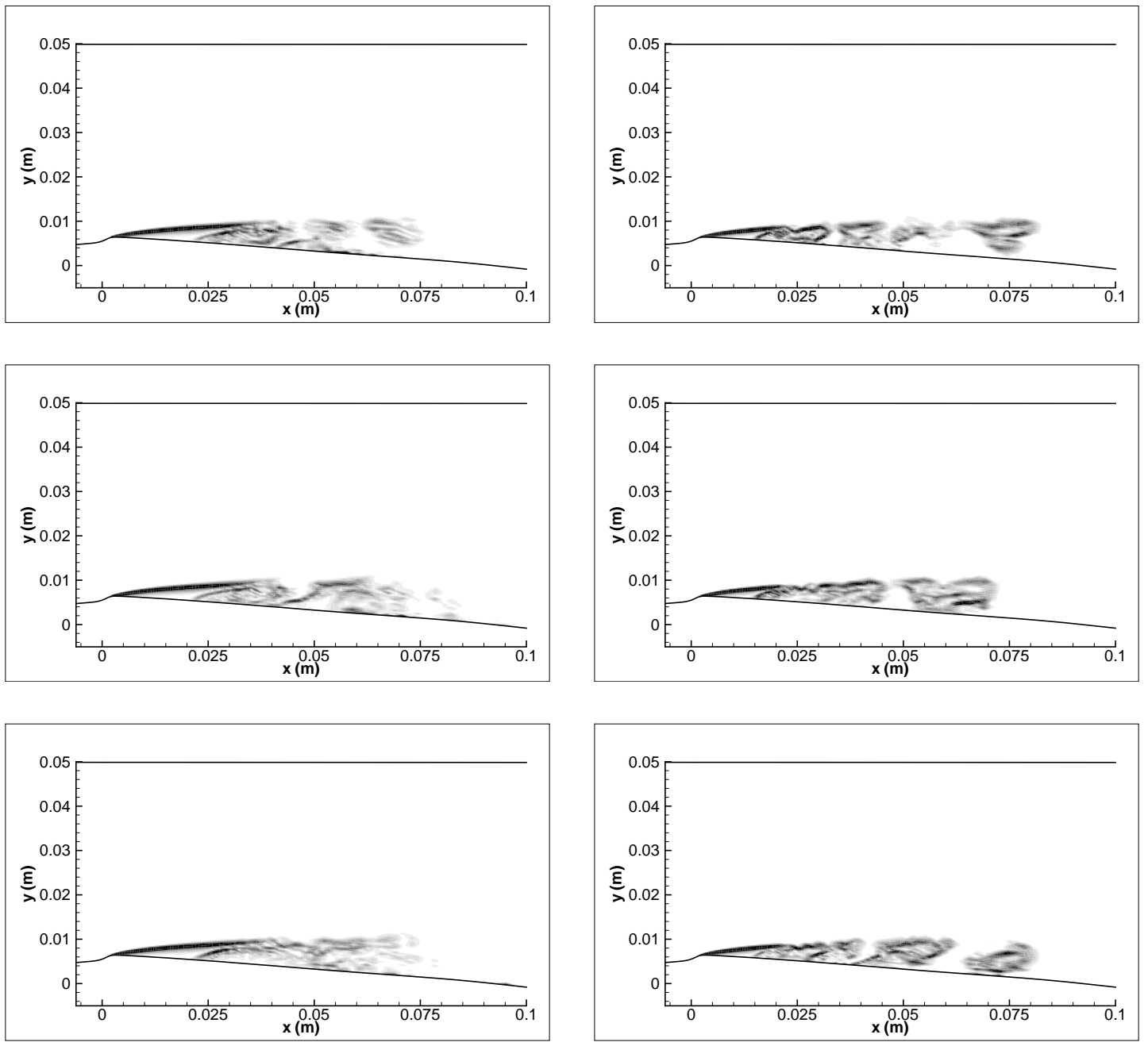

Figure 25: Contours of the density gradient modulus at three instants, KO Reboud $n=4$ (left) and $n=10$ (right), $4^{\circ}$ Venturi. 

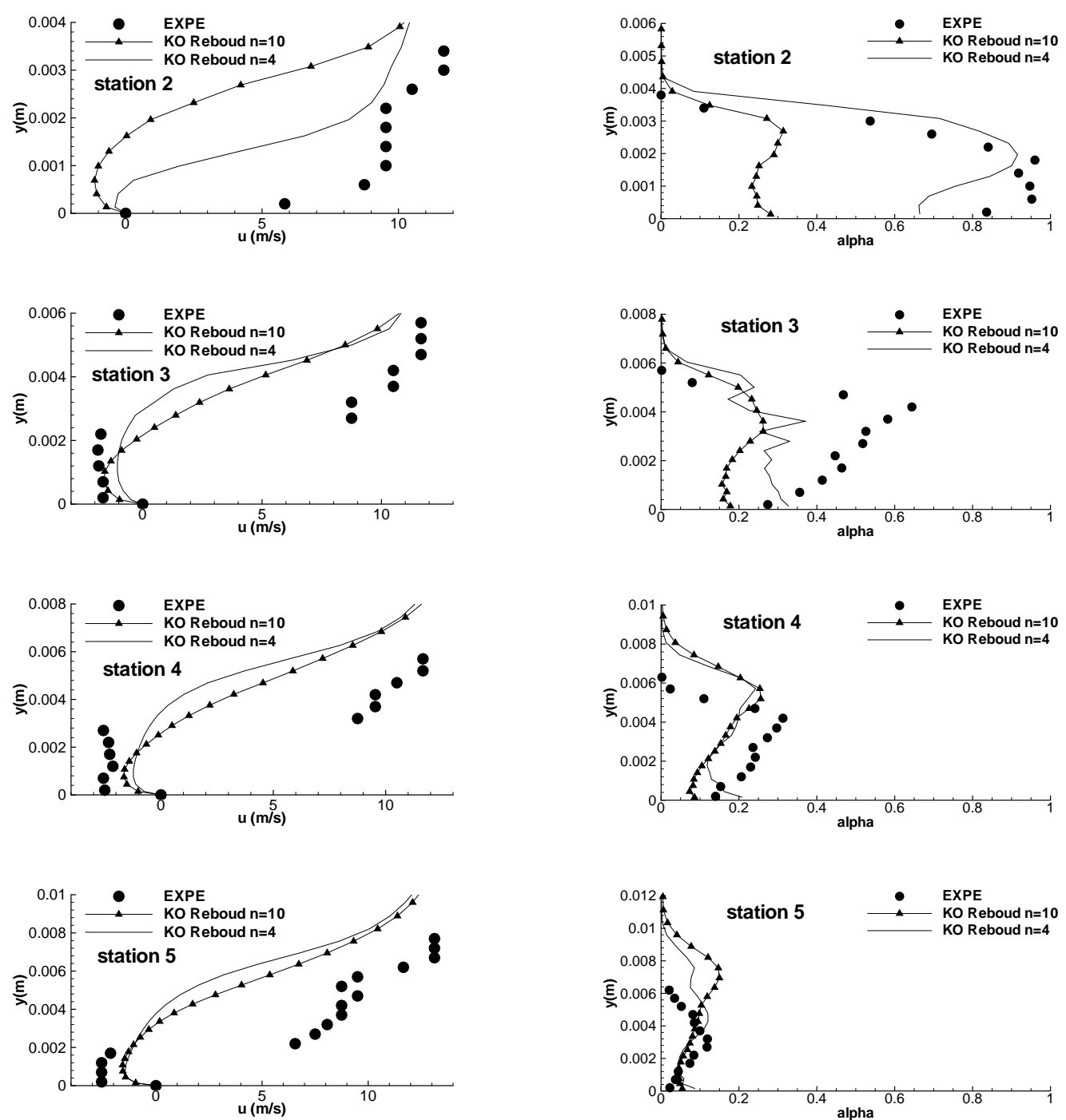

Figure 26: Time-averaged velocity (left) and void ratio (right) profiles from station 2 to 5, KO Reboud model, $4^{\circ}$ Venturi. 


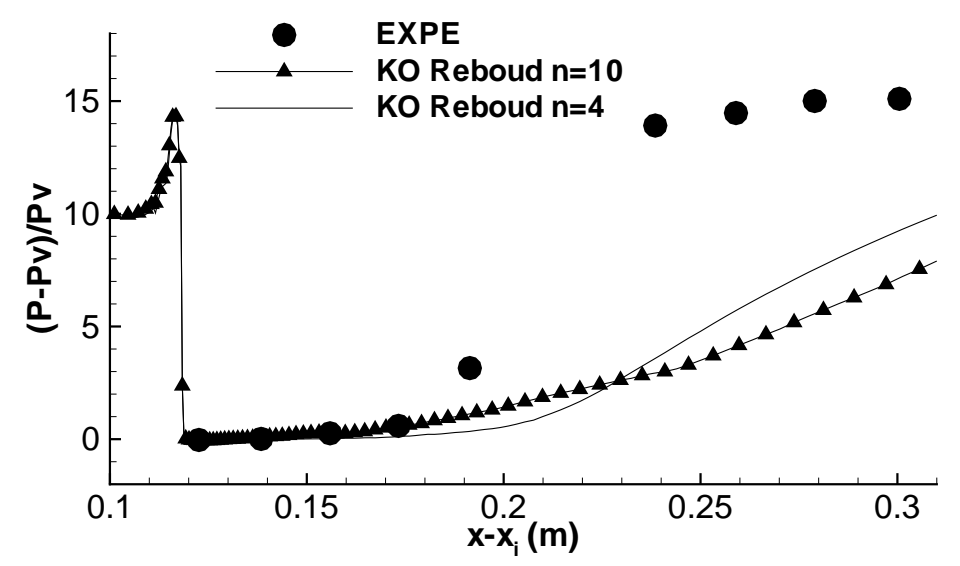

Figure 27: Dimensionless time-averaged wall pressure evolution, KO Reboud models, $4^{\circ}$ Venturi. 


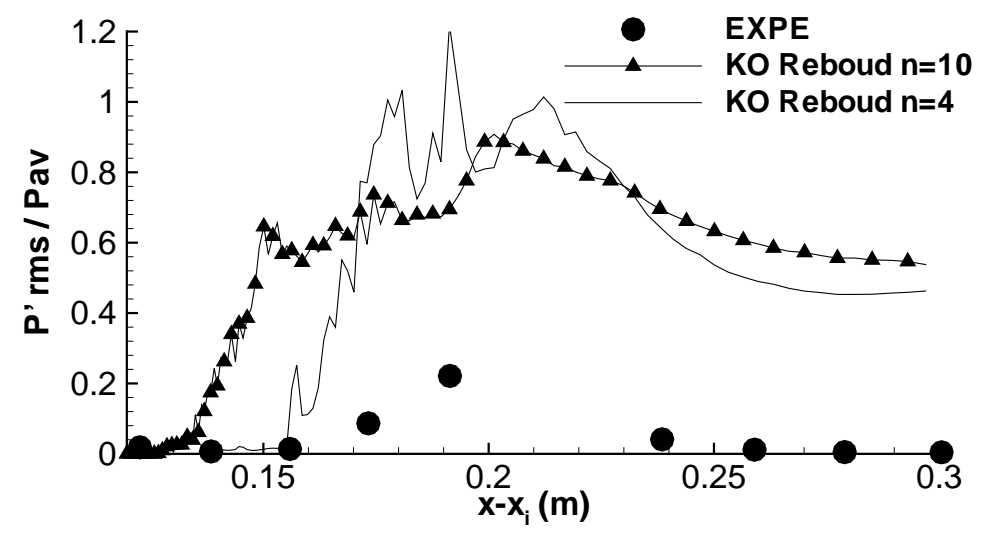

Figure 28: RMS wall pressure fluctuations, KO Reboud models, $4^{\circ}$ Venturi. 

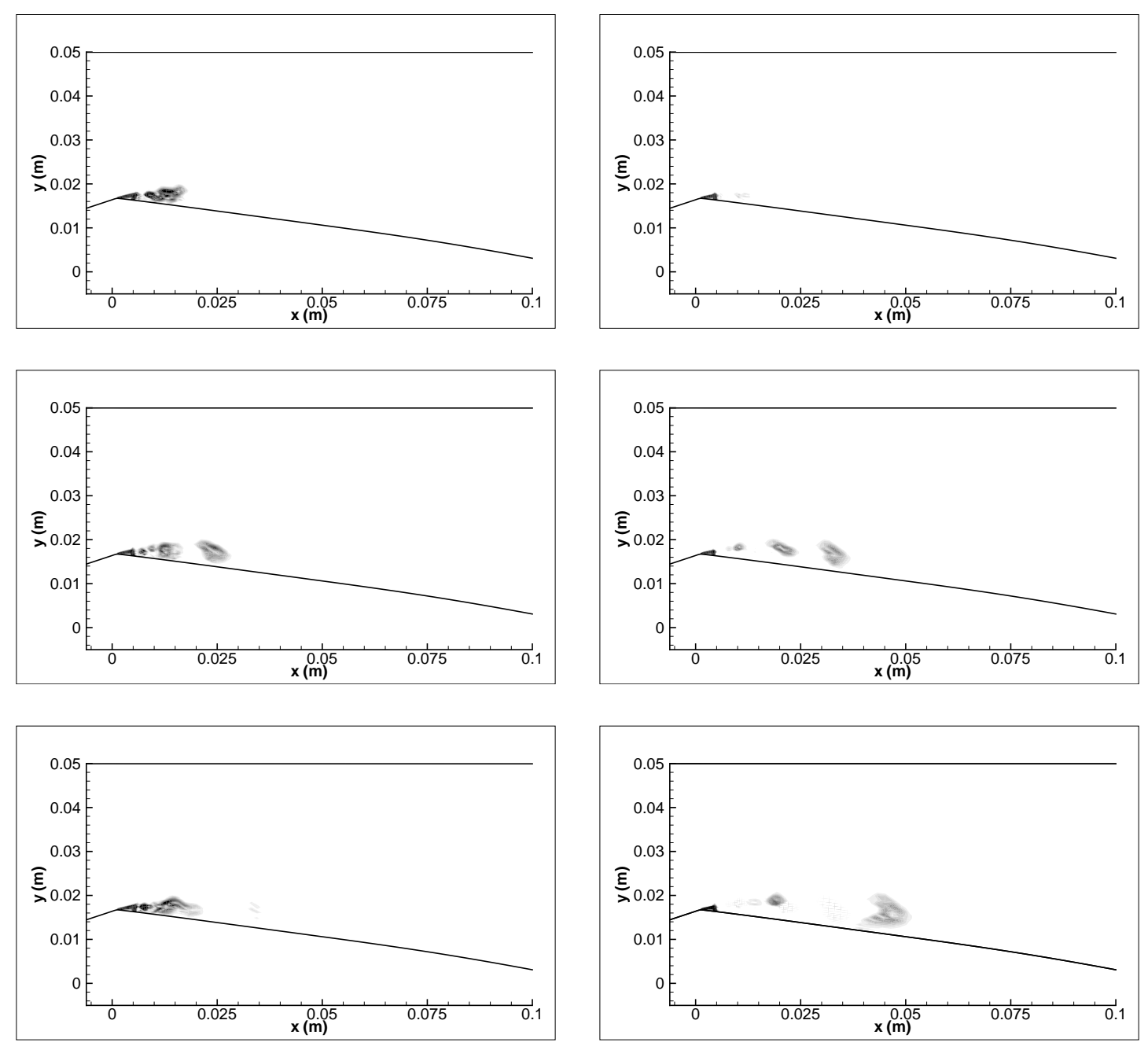

Figure 29: Contours of the density gradient modulus during one period, KO Reboud $n=4$ (left) and $n=10$ (right), $8^{\circ}$ Venturi. 

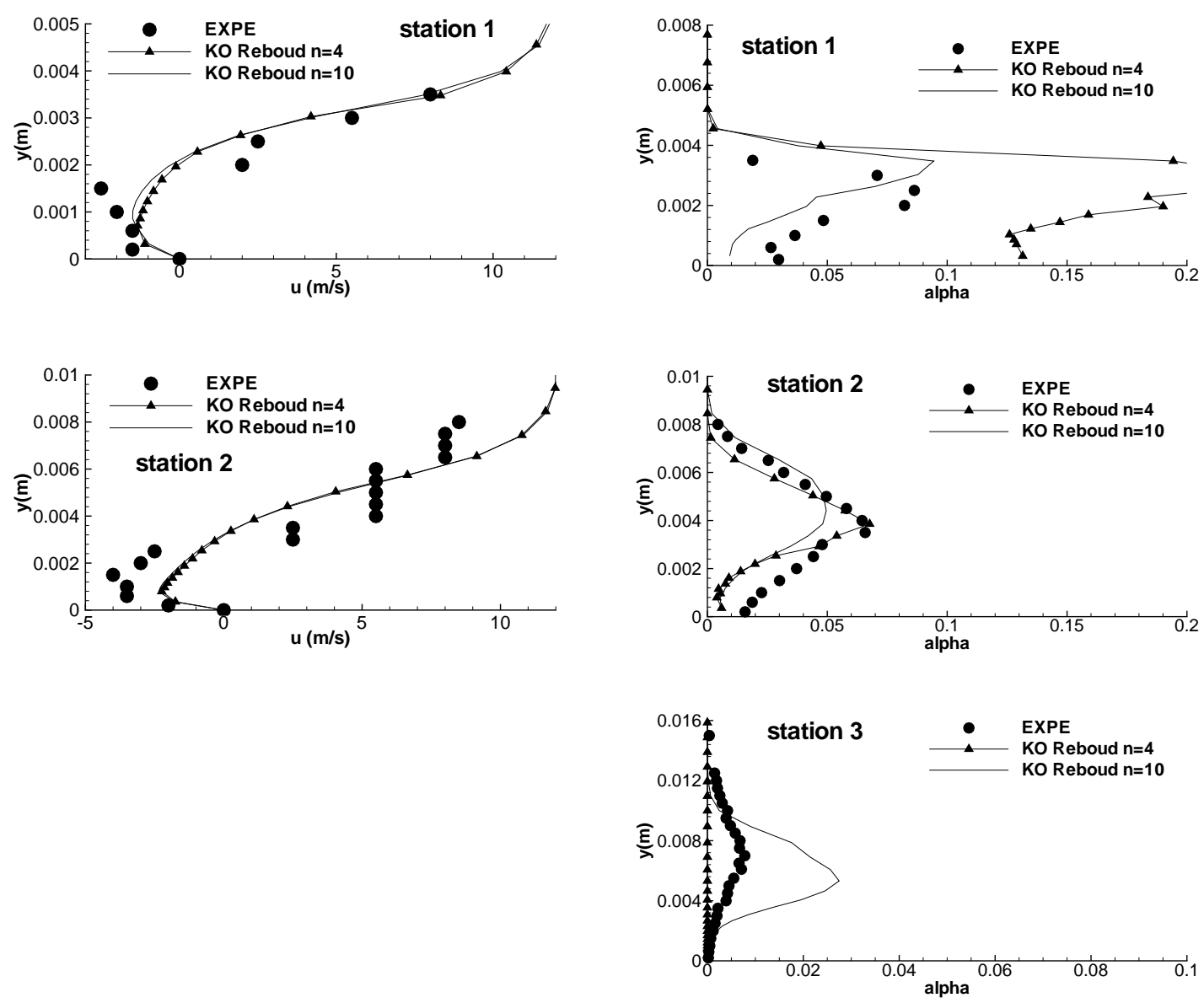

Figure 30: Time-averaged velocity (left) and void ratio (right) profiles from station 1 to 3 , KO models, $8^{\circ}$ Venturi. 


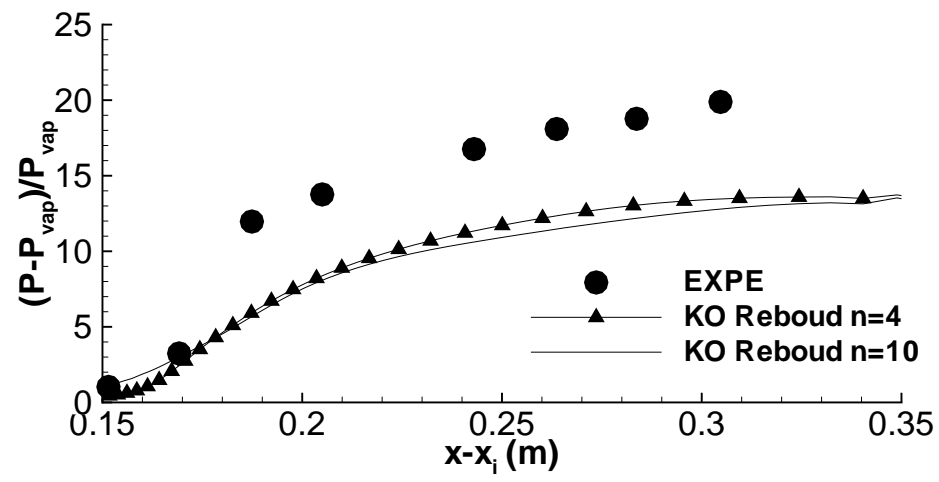

Figure 31: Dimensionless wall pressure evolution, KO Reboud model, $8^{\circ}$ Venturi. 